\title{
Dos ámbitos paleogeográficos para el Paleozoico Superior de los Alpes Cárnicos. Reflexiones sobre la Mesogea pérmica
}

\author{
Ion Argyriadis ${ }^{(1)}$ y Daniel Vachard ${ }^{(2)}$ \\ (1) Cabinet de Géologie Argyriadis, 975 chemin Pré de Caune \\ 83740 La Cadière d'Azur, Francia. \\ ion@argyriadis.net
}

(2) Daniel Vachard, 1, rue desTilleuls, 59152 Gruson, Francia. daniel.vachard@free.fr

\begin{abstract}
RESUMEN
En los Alpes Cárnicos, dos ámbitos paleogeográficos están en contacto tectónico. El ámbito de Zollner See es alpídico y en posición tectónicamente baja; el ámbito de Auernig es dinárico y en posición alta y flotante. El ámbito de Zollner See, autóctono, se caracteriza por un edificio varisco, que culmina en el Kasimoviense superior, y es transgredido en discordancia angular por formaciones detríticas rojas de tipo Gröden, y a continuación por calizas y dolomías yesíferas con Bellerophon. Este ámbito se encuentra sistemáticamente afectado por una esquistosidad verdadera de dirección $110^{\circ}$. El ámbito de Auernig presenta en su base el Gzheliense y alcanza, sin interrupciones estratigráficas significativas, hasta el Pérmico inferior alto, representado por las calizas deTrogkofel; estas últimas se encuentran dominadas en discordancia erosiva por esquistos rojos bastante similares al Gröden, pero que difieren por su espesor, mucho menor, como por su textura y composición. A techo de la sucesión se localiza una formación con Bellerophon de facies mucho más pelágica que su equivalente del ámbito de Zollner See, sobre la que se sedimentó el típico Tríásico de los Alpes Meridionales. Fuera de los Alpes Cárnicos, el ámbito de Zollner See se puede identificar en laToscana septentrional, Islas Baleares (Menorca) o los Pirineos septentrionales, y sobre todo en la Cordillera Cantábrica, que presenta una serie casi idéntica a la de Zollner See. El ámbito de Auernig puede encontrarse en la Toscana meridional y la Isla de Elba.
\end{abstract}

Palabras clave: Alpes Cárnicos, Mesogea pérmica, Nuevos ámbitos, Paleogeografía, Paleozoico superior.

\section{Two paleogeographic realms in the Late Paleozoic of the Carnic Alps and some reflections on the Permian Mesogea}

\begin{abstract}
In the Carnic Alps, two paleogeographic realms are in tectonic contact. The Zollner See realm is Alpidic and in a tectonically low position; the Auernig realm is Dinaric and in a high, floating position. The autochthonous Zollner See realm is characterized by a Variscan core, which culminates in the late Kasimovian, and is unconformably overlain by red detrital strata of Gröden-type, and then by gypsiferous limestone and dolomites with Bellerophon. Systematically, this realm is affected by a true schistosity with a direction of $110^{\circ}$. The Auernig realm has a Gzhelian basal part, and reaches, without noticeable stratigraphic gaps, the late Early Permian, with the Trogkofel Limestone; this latter is overlain, in erosional unconformity, by red shales relatively similar to the Gröden, but which differ by its lesser thickness as well as its texture and composition. At the top of the succession, a formation with Bellerophon, deposited in deeper environments than its equivalent Zollner See realm, is overlain by a typical, southern Alpine Triassic. Beyond the Carnic Alps, only the Zollner See realm can be identified in northern Tuscany, the Balearic islands (Menorca) and the northern Pyrenees, and especially in the Cantabrian Cordillera, which displays very similar a series to that of the Zollner See realm. The Auernig realm can be found in southern Tuscany and the island of Elba.
\end{abstract}

Keywords: Carnic Alps, Late Paleozoic, new realm, Paleogeography, Permian Mesogea. 
Ion Argyriadis y Daniel Vachard, 2020. Dos ámbitos paleogeográficos para el Paleozoico... Boletín Geológico y Minero, 131 (4): $795-815$

\section{ABRIDGED ENGLISH VERSION}

Since the 19th century the Carnic Alps have traditionally been a key area for Upper Paleozoic investigations, due to the alternation between fossiliferous marine strata and fossiliferous continental strata. The structural interpretation, which has largely dominated since Geyer $(1903,1911)$ as well as Vinassa de Regny (1910) and Gortani (1912), is that of a Variscan tectonized frame, transgressively and unconformably overlain by Upper Paleozoic deposits. This dominant idea has been accepted until now. It is worth noting that regional geological studies concerning the area were numerous and excellent, but that, gradually, especially from the end of the last century, they were carried out by sedimentologists or biostratigraphers, though very competent in their own specialities, but who, however, have often used and abused of the concept of "formation", to the detriment of the stratigraphical characteristics of a precise dating and, as a consequence poorly contributed to the understanding of the tectonic structure.

Termier (1922) was the first author, who concluded that the Late Paleozoic was not preserved in situ but corresponded to an Alpine thrust, that of the Alpides upon the Dinarides. It is noteworthy that Heritsch (1936) had already perceived and detected tangential accidents "unquestionably Alpine in age" in this Upper Paleozoic, but he considered them to be a local element of analysis.

Our investigations in these areas indicate the presence of two main types of Paleozoic sequences, which are part of two tectono-sedimentary terranes, which differ in their stratigraphy as well as their geodynamic evolution; one is Alpidic, the other Dinaric. Their locus typicus are found in the Carnic Alps, because the sequences are more complete there and their relationships more explicit, but also because this area was accurately studied for a long time. In the Carnic Alps, these two tectono-sedimentary terranes or realms only display tectonic contacts. The first of these two realms, therefore Alpidic and located below, is called here the Zollner See realm, and the second one, Dinaric and located higher in floating position, is called the Auernig realm. These new names differ from numerous lithostratigraphical names for this area, such as the Auernig Formation or the Auernig Group.

The autochthonous Zollner See realm is characterized by a Variscan core, topped by a fossiliferous Upper Carboniferous culminating a priori in upper Kasimovian strata, unconformably overlain by continental red beds of Gröden type and, then, by gypsiferous limestone "with Bellerophon". One of the fundamental characteristics of this realm is that, unlike the Auernig realm, it is systematically affected by a true schistosity with a direction of $110^{\circ}$. In the Carnic Alps, the Zollner See realm crops out, from west to east, from the Zollner See area to the Monte Cordin and Passo del Cason di Lanza, and, then, it is found under the form of small transgressive outcrops located on a karstic relief in the Rosskofel Massif (Monte Cavallo di Pontebba). More to the south, it is assimilated with the Upper Paleozoic of the Forca Pizzul area.

The Auernig realm, tectonically floating on the Zollner See realm, begins with Gzhelian strata and reaches, without significant gaps, the late Early Permian (Artinskian/Kungurian) of the Trogkofel limestone; this latter is incised by a monogenic breccia; and both are disconformably overlain by red shales which differ from the Gröden by their lesser thickness as well as texture and composition; these red shales are in turn overlain by a formation with Bellerophon with a facies that is more pelagic than its equivalent in the Zollner See realm. This formation with the Bellerophon of the Auernig realm is overlain by a typical south-alpine Triassic, which crops out either in continuity, or to the east, up to the area of the Gartnerkofel Triassic massif, with a tectonic contact. More to the east, up to the Tarvisio area, the sparse and tectonically isolated outcrops can be assigned with difficulties to each realm.

The contact between both realms is only tectonic, and is underlined by a belt of Upper Permian deposits (Gröden and Bellerophon formations), easy to follow from the Monte Cordin to Passo del Cason di Lanza, but then they are hidden in the north of the Devonian massif of Rosskofell. Nevertheless, the two realms are present, in tectonic contact, in the south of Nassfeld (Passo di Pramollo), although this contact is only marked by a tectonic breccia, since the Upper Permian is laminated and possibly only inferred from sulphuric springs.

The second part of this paper, according to the available number of pages, is devoted to a brief investigation of the possible paleogeographic distribution of both realms in other European areas, which are defined here.

Extending to the east and south-east, there is a large area where both types of realms are found, especialIy the Auernig realm, the Permian Mesogea, which, progressively, was open to the south-east. However, it is possible to identify the Zollner See realm in the para-autochthonous of the Bükk Mountains (Hungary), which 
Ion Argyriadis y Daniel Vachard, 2020. Dos ámbitos paleogeográficos para el Paleozoico... Boletín Geológico y Minero, 131 (4): $795-815$

crops out within the Pannonian Basin, as well as in western Serbia, in Chios Island (Greece), in the Gökdag series in Anatolia (Turkey), etc.

Westwards, the Auernig realm is difficult to identify. It probably exists in southern Tuscany, in the island of Elba (Italy), etc. Inversely, the Zollner See realm there is more easily identifiable; peculiarly, in northern Tuscany (Italy), the Balearic islands (Minorca, Spain), in the northern Pyrenees (France), etc. Furthermore, the Cantabrian Cordillera (Spain) exhibits the clearest and most complete example of a series almost similar to that of Zollner See. The Cantabrian Cordillera indeed includes (see Villa and Sánchez de Posada 2009, Villa 1989, Villa et al. 2003, Sánchez de Posada et al. 1996, etc.) a Carboniferous series from the Tournaisian to the Kasimovian, constituted by detrital sequences (shales, sandstones, turbidites) and also limestone. The series contains marine fossils as well as terrestrial plants. Marine fossils are brachiopods and ammonoids for the Early Carboniferous and fusulinids for the Upper Carboniferous (Villa et al. 2003). In the Picos de Europa Unit the highest stratigraphic levels were found, namely the upper Kasimovian (Villa et al. 2003). In all the localities, the detrital red Permian is transgressive regionally upon the Carboniferous series, incising the substrate and with an unconformity. It is evident that the similarity with the sequences of the Zollner See is important. On the other hand, the cited authors highlighted the correlation with the Carnic Alps, but without the difference between the two realms that has been established in this paper.

We can define and justify the existence of an end-Paleozoic realm that surrounds the Hercynian continent along the margin of the post-Hercynian Mesogea/Paleotethys. This latter is characterized by a marine Paleozoic series, especially by a marine Carboniferous affected by terrestrial imputs with plants - and with carbon -, which extends up to the latest Kasimiovian, and possibly to the earliest Gzhelian, and even to the Asselian (in Zollner See according to Heritsch 1936). This unit is marked, at least in the Carnic Alps, by a true schistosity of $110^{\circ}$, transgressively overlain by a red Permian with a strong unconformity, which in turn, evolves to carbonates and evaporites. Upward, it is topped by a troncature surface in the sense of Ellenberger (1967). This realm differs from that of the Auernig, in the Carnic Alps, which does not show a substrate known in situ because it is tectonically floating, and begins with the Gzhelian, and because the marine series reaches, with terrigenous imputs, at least the uppermost Lower Permian, with some reefal intercalations. The transgression of a red Permian also exists, but the Saalian unconformity only corresponds to a local disconformity, and, there the Late Permian is more carbonated than evaporitic, and is overlain by a marine Mesogean Triassic.

Finally, we would like to pay tribute to the great geologist Pierre Termier, who was the first to mention the superposition in the Carnic Alps "of the Dinarides upon the Alpides". Such a superposition probably results from the Cretaceous orogenesis (Argyriadis 1974, 2016) that Suess, with excellent intuition (1892), called the "posthumous Altaids". The last problem to solve is to know which part of this orogenesis and the Variscan are represented in the Carnic Alps.

\section{Introducción}

La región situada entre el nordeste de Italia, el sur de Austria y el noroeste de Eslovenia (Friuli, Alpes Cárnicos, Karawanken y Alpes Julianos) resulta especialmente interesante bajo el punto de vista de sus terrenos del Paleozoico superior (Figura 1). En ella se observan alternancias de capas fosilíferas de facies marinas con otras capas fosilíferas de facies continentales, particularidad que ha llamado la atención de los científicos desde mediados del siglo XIX. Las investigaciones se sucedieron a un ritmo creciente $y$ el volumen de bibliografía sobre el tema es enorme. A finales del siglo XX y principios del XXI, los estudios trataban principalmente sobre el análisis bioestratigráfico permitiendo un mejor conocimiento de las series geológicas. Siguiendo una moda procedente de los petroleros americanos, se definieron diversas "formaciones" y "grupos" litoestratigráficos (Pontebba Group, Pramollo Group, Hochwipfel Formation, Bombaso Formation, etc.). Este procedimiento, si bien resulta útil, tiende a ocultar las relaciones estructurales $y$, en todo caso, no facilita su comprensión. Así pues, desgraciadamente, el análisis tectónico - sin mencionar la interpretación estructural - no siguió la misma progresión. Este trabajo tiene como objetivo fundamental examinar las cuestiones de paleogeografía regional así como las de las partes respectivas de la tectónica herciniana y de la tectónica alpina.

Dos grandes tipos de series del Paleozoico superior, que existen en estas regiones, pertenecen a ámbitos que difieren tanto por su estratigrafía como por 
su evolución geodinámica. El locus typicus para cada uno de ellas se localiza en los Alpes Cárnicos, porque allí es donde se encuentran las series más completas y también, por cuestiones históricas, esta región ha sido estudiada en detalle desde hace tiempo. La interpretación estructural dominante desde Geyer (1903, 1911), Vinassa de Regny (1910) o Gortani (1912) fue la de un edificio tectonizado herciniano, recubierto en transgresión y discordancia angular por un Paleozoico superior casi tabular iniciado en el Carbonífero superior, seguido de una serie pérmica y, como colofón, por el Triásico inferior. Dicho esquema ha sido adoptado hasta la actualidad en mayor o menor grado. Sin embargo, Termier (1922) fue el primer investigador en ponerlo en duda, afirmando que de hecho se trataba de "un corrimiento de edad alpina de los Dinárides sobre los Alpides". Más tarde, Heritsch describió en su monografía (1936), pero a menor escala, una tectónica incontestablemente alpina en esta "cobertura" paleozoica superior, incluyendo una componente tangencial.

Uno de los dos ámbitos fue denominado como ámbito de Auernig-Gartnerkofel (Argyriadis, 1968, 1970). La serie empieza en el Carbonífero superior (Gzheliense superior-Orenburgiense) descrito por primera vez por Stache (1872) y cuyo perfil detallado fue efectuado por Frech (1894) bajo el nombre de "estratos de
Auernig". Sobre ese Carbonífero se depositó un Pérmico casi completo, con una proporción importante de estratos carbonatados. En su parte alta, se encuentra un Scytiense más carbonatado que el de los Alpes Meridionales y finalmente, tras un nivel conglomératico ("Brecha de Uggowitza" o "conglomerado de Richthofen"), se pasa a un Anisiense menos grueso que el del ámbito Subalpino y al Ladiniense de facies "Schlerndolomit". El contacto de la base de esta serie siempre es tectónico. Se designa dicho ámbito bajo la forma abreviada de ámbito de Auernig que no debe confundirse con la Formatión Auernig de las publicaciones bioestratigráficas recientes, cuya parte inferior pertenece a otro ámbito paleozoico superior presente en la región. Este segundo ámbito se caracteriza por una serie geológica que empieza con la transgresión del Carbonífero superior (Moscoviense terminal-Kasimoviense inferior) sobre terrenos paleozoicos muy diversos (Silúrico con graptolitos, Devónico arrecifal, o "estratos de Hochwipfel"). En el Pérmico, ésta serie geológica resalta por la irregularidad de sus perfiles y viene marcada por todas partes por la transgresión en discordancia angular y erosiva del Pérmico superior en forma de los estratos de Gröden y, por encima de los estratos con Bellerophon, muy similares en facies y espesor a los de los Alpes Meridionales. El Pérmico

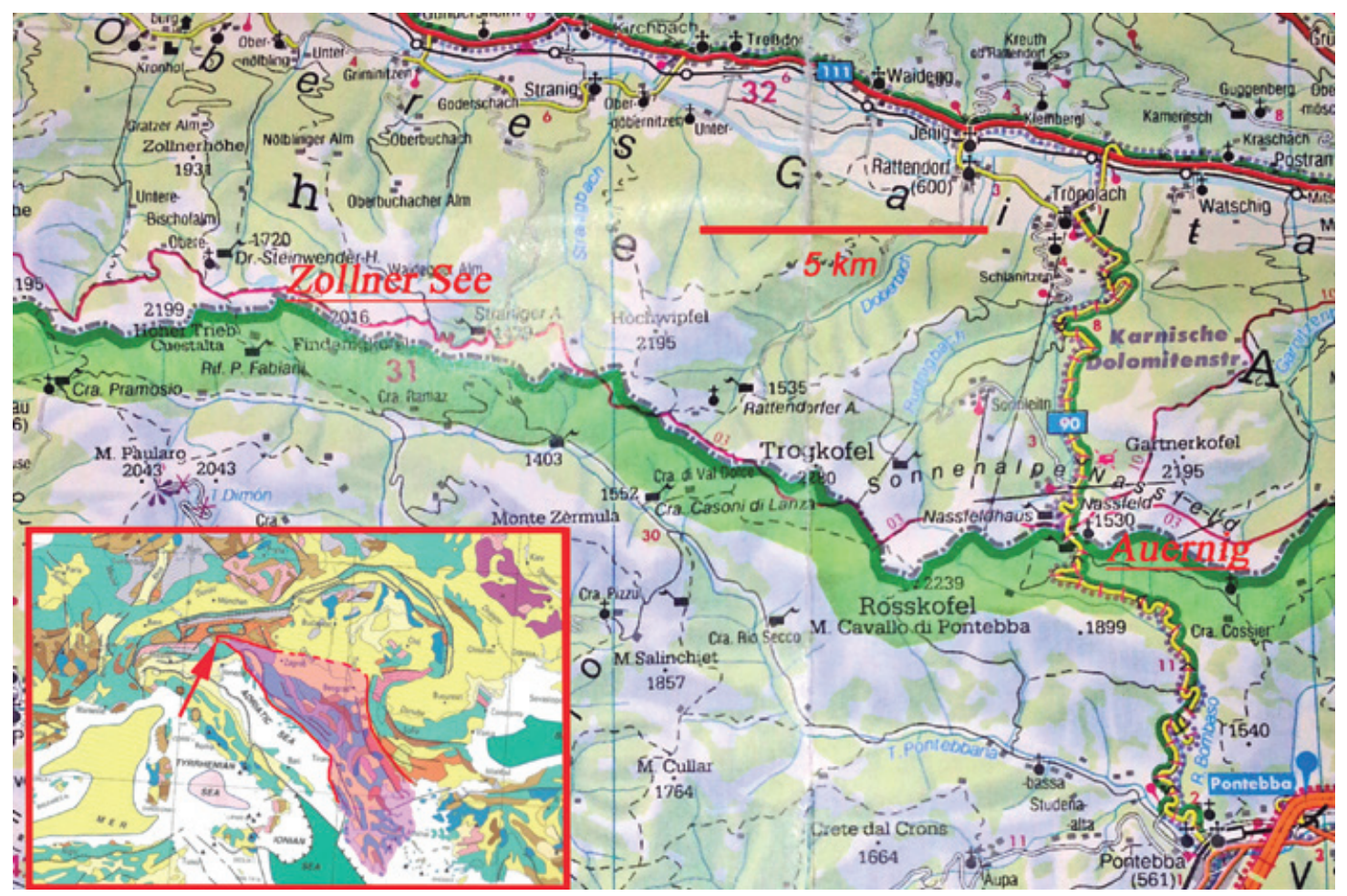

Figura 1. Mapa de localización. Abajo y a la izquierda, esquema geológico con la situación de Alpides y Dinárides (en violáceo) y la ubicación del área estudiada (flecha roja).

Figure 1. Location map; with a geological sketch (bottom, left) showing the location of the Alpides and the Dinarides (coloured in magenta) and the studied area (red arrow). 
inferior falta, exceptuando un minúsculo afloramiento de caliza asseliense, situado al SW de Zollner See indicado por Heritsch et al. (1933, p. 177) y Heritsch (1935 p. $367 ; 1936$ p. 82). Esta serie se encuentra en toda la región de los Alpes Meridionales, con variaciones locales como la ausencia de algunas zonas del Carbonífero superior y la transgresión directa del Pérmico superior rojo sobre el Carbonífero medio o incluso el Paleozoico inferior. Argyriadis (1968) describió la serie bajo el nombre de "serie de tipo Monte Dimón", que Mariotti (1972) llamó más tarde "serie de la unidad de Straniger Alm". El estudio detallado, desde el 1999, ha mostrado que su expresión más completa se encuentra entre el Zollner See y el Monte Cordín, y por tanto, se propone el término "serie de tipo Zollner See", para definir el ámbito del mismo nombre.

Las unidades correspondientes a los ámbitos antes mencionados se encuentran actualmente en contacto exclusivamente tectónico. La unidad del ámbito de Auernig es la más alta tectónicamente, mientras que la de Zollner See se encuentra sistemáticamente en posición tectónicamente baja. Esta relación fundamental puede estar encubierta localmente por fragmentaciones accidentales posteriores al transporte, pero se encuentra perfectamente expuesta en toda la región que va desde Zollner See hacia el este en dirección a Straniger Alm, Monte Cordin (Straniger Kopf), Schulterkofel, Pian di Lanza (Lanzenboden), Casera Valbertad Alta y, más al este, hasta el basamento mismo del Monte Auernig.

\section{Las dos sucesiones del Paleozoico Superior}

\section{El ámbito de Zollner See}

Este ámbito debe ser considerado como un autóctono en relación con el contexto post-varisco y se adopta una edad varisca para los mantos definidos por von Gaertner (1931) en dicha región. No se deben confundir el ámbito de Zollner See y la Zollner Formation. Esta última se compone de "liditas, radiolaritas y esquistos piritosos" que van "del Devónico medio al Carbonífero inferior" (Schönlaub 1985). El ámbito en cuestión se caracteriza por la existencia de un Carbonífero medio de facies Culm ("estratos de Hochwipfel" de los geólogos austríacos) transgresivo en diferentes substratos con discordancia angular y una muy fuerte discordancia erosiva, sobre la que se dispone un Paleozoico superior incompleto, iniciado en el Kasimoviense, y con un Pérmico que sólo se encuentra completo a partir de la transgresión de los estratos rojos de Gröden, con una discordancia erosiva muy importante. Por encima, los estratos con Bellerophon del Pérmico superior son dolomíticos y evaporíticos, dominados por un Triásico basal de tipo "Seis" que prosigue mas lejos hacia el Sur, por el Anisiense, Ladiniense y finalmente por el Cárnico.

\section{Estratigrafía}

El corte más completo de la serie estratigráfica de dicho ámbito se encuentra justamente en la región de Zollner See y se extiende desde los alrededores de ese lago hasta los del Monte Cordin, donde empieza a estar dominada tectónicamente por las formaciones del dominio de Auernig. Este corte fue indicado por Pölser (1969) y publicado por Mariotti (1972). La sección más reveladora se localiza a la salida del lago que se halla en su extremo oeste. Para estudiarla, hay que penetrar por la parte alta del aliviadero en donde forma una cascada y seguir durante algunos metros el curso del arroyo. Esta sección de difícil acceso presenta puntos de discusión en lo que respecta a su sustrato. De base a techo, se observan:

1. El sustrato está representado por esquistos ampelítosos negros, accesibles a aproximadamente $20 \mathrm{~m}$ bajo los estratos carboníferos transgresivos. Dichos esquistos contienen graptolitos: Monograptus suessi Perner y $M$. riccartonensis Lapworth que permiten datar ese nivel del Wenlock (determinación de Waterlot, en Mariotti 1972). Los esquistos se hallan fuertemente plegados. Se trata del Silúrico "de facies Bischofalm" según la nomenclatura austríaca.

2. La sección esta semicubierta en algunos metros, pero el primer nivel transgresivo, estimado como tal a causa de su buzamiento horizontal así como de su facies, está constituido por esquistos "de bolas" y por brechas poligénicas con elementos angulosos centimétricos a decimétricos de lidita, cuarzo y arenisca.

3. Por encima, se encuentran algunos metros de pelitas esquistosas que contienen cantos rodados aislados, redondeados, de liditas. Estos niveles pertenecen muy probablemente, teniendo en cuenta sus facies, a los "estratos de Hochwipfel", cuya edad, muy debatida desde inicios del siglo XX, se confirma como del Mississíppico superior al Pensilvánico inferior (Namuriense para Heritsch 1936 o Serpukhoviense para Krainer y Vachard 2015).

4. Tras atravesar condiciones de observación escasas, se encuentran 1 a 2 metros de arenisca esquistosa con vetas lenticulares de microbrechas con elementos de lidita.

5. Directamente por encima y en continuidad, existen 2 metros de calizas areniscosas que contie- 
nen fusulinidos pero también elementos angulosos de lidita y cuarzo. Entre los fusulinidos se distinguen Protriticites pramollensis (Pasini), Quasifusulinoides intermedia (Rauzer-Chernousova y Rozovskaya), Ozawainella angulata (Colani) y Wedekindellina sp. (in Pasini 1963). En este nivel, se encuentran también presentes algunas algas como Anthracoporella spectabilis Pia. Se trata de una asociación del Kasimoviense basal.

6 . Sobre el nivel precedente se hallan $50 \mathrm{~cm}$ de pelitas oscuras, esquistosas

7. Areniscas finas en bancos gruesos

8. Areniscas psamíticas esquistosas de color gris rojizas.

El mapa geológico austriaco indica en varios lugares un nivel transgresivo representado por "una brecha de liditas, conglomerados, caliza y esquistos". Krainer (1992) dató ese nivel entre el Moscoviense terminal y el Kasimoviense basal (en base a los Quasifusulinoides quasifusulinoides, Protriticites ovatus, Fusiella lancetiformis, Ozawainella, Pseudotriticites, Staffella y Schubertella). Un hecho sobresaliente es que dicha serie se ve afectada por una verdadera es-

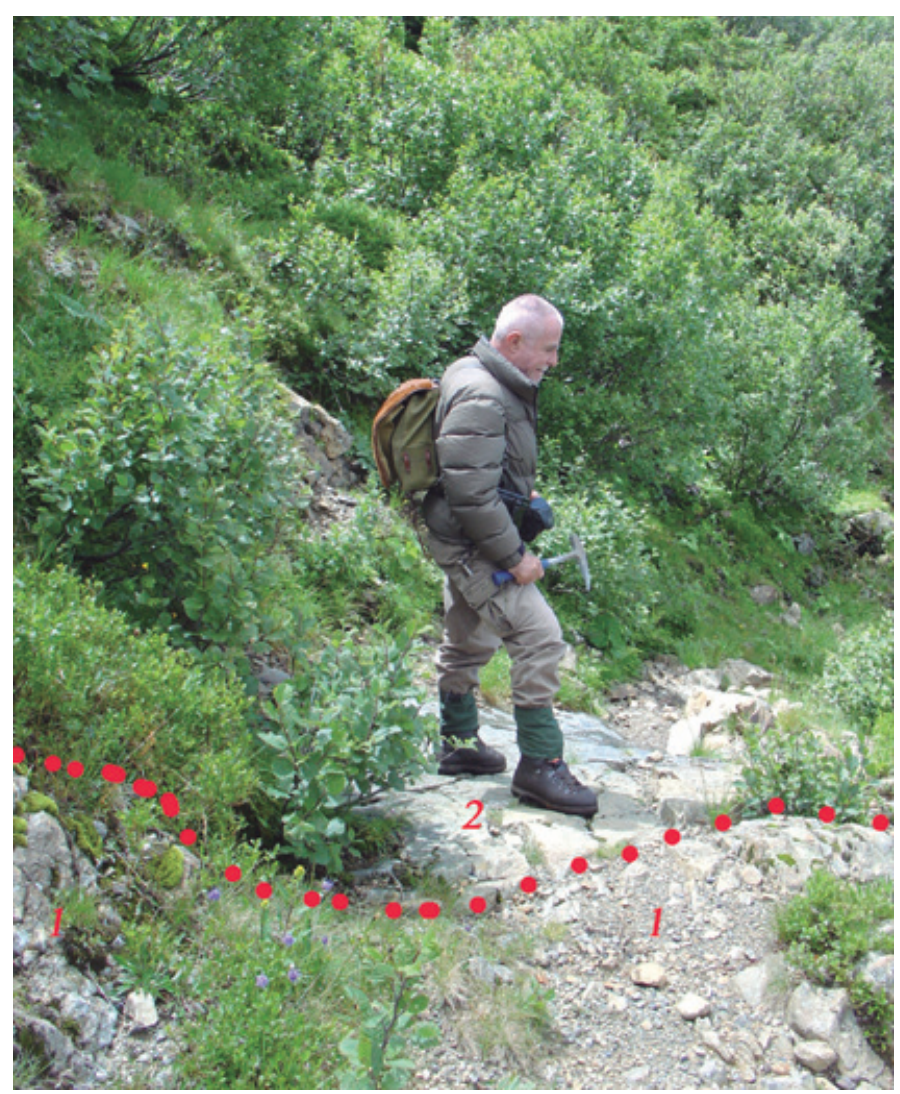

Figura 2. Brecha con elementos de lidita y de arenisca (1) transgresiva sobre areniscas pelíticas finas (2, véase en el texto).

Figure 2. Breccia with pebbles of lydite and sandstone (1), transgressive on fine, pelitic sandstones (2, explanations in the text). quistosidad con una dirección de $110^{\circ}$. Esto atañe a todos los terrenos del ámbito de Zollner See como veremos más adelante. Localmente, conviene añadir las observaciones siguientes:

En el sendero que va desde el refugio "Zollner Hütte" hacia el lago Zollner See, se observa la transgresión de una brecha grosera con elementos decimétricos a pluridecimétricos angulosos de lidita negra y de arenisca subyacente (Figura 2, 46 $36^{\circ}$ 09.24" N

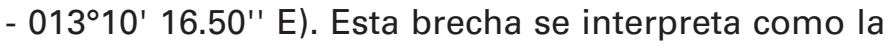
primera transgresión, la de la Formación Hochwipfel, sobre el sustrato varisco.

Este nivel es transgresivo directamente sobre areniscas pelíticas y cuarcíticas claras, muy finas, con elementos submilimétricos de cuarzo y, más raramente, de lidita. Es posible que se trate de Silúrico. Sin duda, cabe señalar que su facies recuerda a la del Ordovícico (Ashgill) de Bischofalm, pero resulta asombroso que en una distancia horizontal de alrededor de $200 \mathrm{~m}$ la erosión haya podido hacer desaparecer toda la base del Silúrico. A este argumento se añade la presencia de finos clastos de lidita, que sólo pueden provenir de las liditas del Wenlockense circundante.

A unos $200 \mathrm{~m}$ al Sur del lago existe un sinclinal con carbonatos muy abierto con inclinaciones muy débiles, Ilamado Colendiaul por los geólogos austríacos, y descrito como uno de los lugares en los que se encuentra representada la transgresión del Carbonífero superior de los "estratos de Auernig". Es necesario indicar que el contacto de la transgresión no es muy claro en afloramiento, teniendo en cuenta las condiciones tectónicas, pero también las condiciones de afloramiento. En la cima de la meseta se vislumbran calizas negras, caliza gris con crinoideos, calizas con pátina amarilla, con crinoideos $y$, en la parte superior, margas calcáreas, con un espesor total de 5 metros aproximadamente. Dichas calizas contienen fusulinidos, entre los cuales Protriticites cf. pramollensis (Pasini) (con una pared que se vuelve keriotecal) y Quasifusulinoides sp., así como el alga Anthracoporella spectabilis $\mathrm{Pia}$, que indican que la edad sigue situándose en el Kasimoviense inferior.

\section{Las áreas de afloramiento}

Este tipo de serie del Carbonífero superior siempre se interrumpe a mitad o a techo del Kasimoviense y más al este, a partir del Monte Cordin (Stranigerkopf), es transgredida directamente por las formaciones detríticas del Pérmico superior de facies Gröden. En un solo lugar, al SO de Zollner See, Heritsch (1936, p. 82 véase supra) indica la presencia de "la caliza inferior con Pseudoschwagerinas" que se apoya normalmente sobre los estratos de Auernig más profundos ("un- 
tere kalkarme Schichtgruppe"). Se trata del Asseliense reposando directamente sobre nuestro Kasimoviense.

El Kasimoviense aflora hacia la vertiente occidental del Monte Cordin, con Rugosofusulina alpina Schellwien, Ozawainella nikitovkensis Brazhnikova y Anthracoporella spectabilis Pia. Es un Kasimoviense superior, sobre el que transgreden areniscas rojas de facies Gröden. La base de la transgresión destaca por un conglomerado rubefactado con cantos pequeños de cuarzo, y por encima un nivel dolomítico. No se observa discordancia angular perceptible. Esta transgresión fue descrita por Mariotti (1972) quién la convirtió en uno de los rasgos distintivos de su "serie de Straniger Alm", a pesar de que era conocida desde tiempo atrás (Geyer 1896 p. 145; Heritsch 1936 p. 141, 142). Estos autores no le atribuyeron la importancia que merece $y$, por ejemplo, no establecieron una distinción entre dicha serie y la del ámbito de Auernig (cf. infra), diferente y mucho más completa.

El Pérmico superior rojo se puede seguir hacia el este, hasta la región situada entre Passo del Cason di Lanza y Pian di Lanza (Lanzenboden). Es transgresivo sobre las formaciones kasimovienses y está cabalgado por el Carbonífero superior del ámbito de Auernig (Gzheliense-Orenburgiense). En el extremo oriental de dicha banda de terrenos pérmicos, éstos últimos se ven completados hacia la parte alta de la sucesión por algunos metros de dolomías y de carniolas, en las que se pueden diferenciar facies claras y otras negras, bituminosas. Dichas formaciones pudieran pertenecer a los "estratos de Bellerophon" y particularmente a su facies "Fiamazza" del ámbito suralpino.

Aquí, la serie del ámbito de Zollner See se encuentra limitada por un contacto anormal, y se halla recubierta por la serie transportada del ámbito de Auernig.

Hacia la base, esta misma serie se muestra transgresiva en la región del Río Malinfier (Marchbach) sobre un sustrato muy erosionado, formado por calizas rosas nodulares con tentaculítidos y conodontos, del Devónico, luego, y un poco más al este, al nivel de la Casera Valbertad Alta, sobre esquistos y liditas negras del Silúrico.

En estribaciones más lejanas, hacia el este, el accidente tectónico del margen norte del Monte Zermula corta el edificio y acaba por ocultar el ámbito de Zollner See, hasta llegar directamente a estar en contacto con el ámbito de Auernig. No obstante, al sur de ese contacto, se encuentran jirones transgresivos dispersos de la serie de Zollner See

En la región de Forca Pizzul, situada al este de Paularo, entre los Montes Pizzul y Salinchiet, sobre la pista que sube hacia la Casera Pizzul y a 1450 m de altitud, se observa una transgresión substancial: el sustrato está compuesto por lavas y tufitas verdes con inclina- ción rígida $\left(60^{\circ}\right.$ hacia el sur), afectadas por fallas "selladas" por la transgresión en discordancia angular muy marcada (inclinación de $20^{\circ}$ hacia el ESE) de 30 $\mathrm{cm}$ de pelitas esquistosas muy finas, $y$ otros $30 \mathrm{~cm}$ de areniscas calcáreas sobre las que se sedimentaron 20 $\mathrm{cm}$ de pelitas y $40 \mathrm{~cm}$ de areniscas de grano fino. Estas formaciones evolucionan hacia arriba y la Casera Pizzul, hacia un conjunto típico del Kasimoviense de ZoIlner See. Se trata de pelitas, esquistos nodulares de color óxido, areniscas micáceas quebradizas, conglomerados de cantos rodados de cuarzo blanco y calizas margosas amarillas con fractura negra, enmarcadas a menudo en negros bituminosos. La parte esquistosa contiene una flora del Westphaliense D (Reichardt 1935) y las calizas contienen “braquiópodos, gasterópodos, y algas del Carbonífero superior" (fide Vinassa de Regny y Gortani 1905). Argyriadis (1978) encontró Ammovertella y Rugosofusulina sp. Lo que pudiera autorizar la atribución de dichos estratos al Kasimoviense del ámbito de Zollner See.

Por encima de la Casera Pizzul y al pasar por la Casera Paluchian hacia la cresta que transcurre entre el Monte Pizzul, al Norte, y el Monte Salinchiet, al Sur, las cosas se complican desde el punto de vista tectónico. Los estratos del Carbonífero superior comienzan a erguirse, e inmediatamente por encima de la Casera Paluchian $\left(46^{\circ} 32^{\prime} 34^{\prime \prime} \mathrm{N}, 013^{\circ} 04^{\prime} 41.18^{\prime \prime} \mathrm{E}\right)$, se encuentra una banda métrica de caliza gris claro con Trocholina (Cordevoliense? muestra AC-99-32) intercalada tectónicamente entre una caliza negra con fusulínidos y, por encima, otros estratos del Carbonífero superior. En la propia cresta, se puede seguir, de norte a sur, une sucesión de escamas, entre las que la más meridional deja entrever lavas del Triásico dominadas por el Ladiniense calcáreo blanco del Salinchiet. No obstante, una de esas escamas, alrededor de un punto elevado de $1740 \mathrm{~m}$, pone de manifiesto la transgresión directa de areniscas rojas de Gröden sobre el Carbonífero superior, a través de una brecha de $50 \mathrm{~cm}$ de espesor con cantos rodados de cuarzo, dolomías y areniscas. Esta transgresión ya había sido indicada (Vinassa de Regny y Gortani, 1905; Argyriadis, 1970, 1978; Mariotti, 1972).

El macizo de Rosskofel (Monte Caballo di Pontebba) está constituido por caliza arrecifal (Devónico a Carbonífero inferior?). Está limitado al norte por un accidente tectónico de inclinación sur, que lo hace chocarse contra los estratos del ámbito de Auernig (Carbonífero superior pasando en su parte alta hacia el Pérmico). Sin embargo, en el interior del macizo se observan varias transgresiones pequeñas de estratos calcáreos y detríticos pertenecientes al Kasimoviense de tipo Zollner See. Todas ellas tienen como característica común estar depositadas sobre un sustrato de 


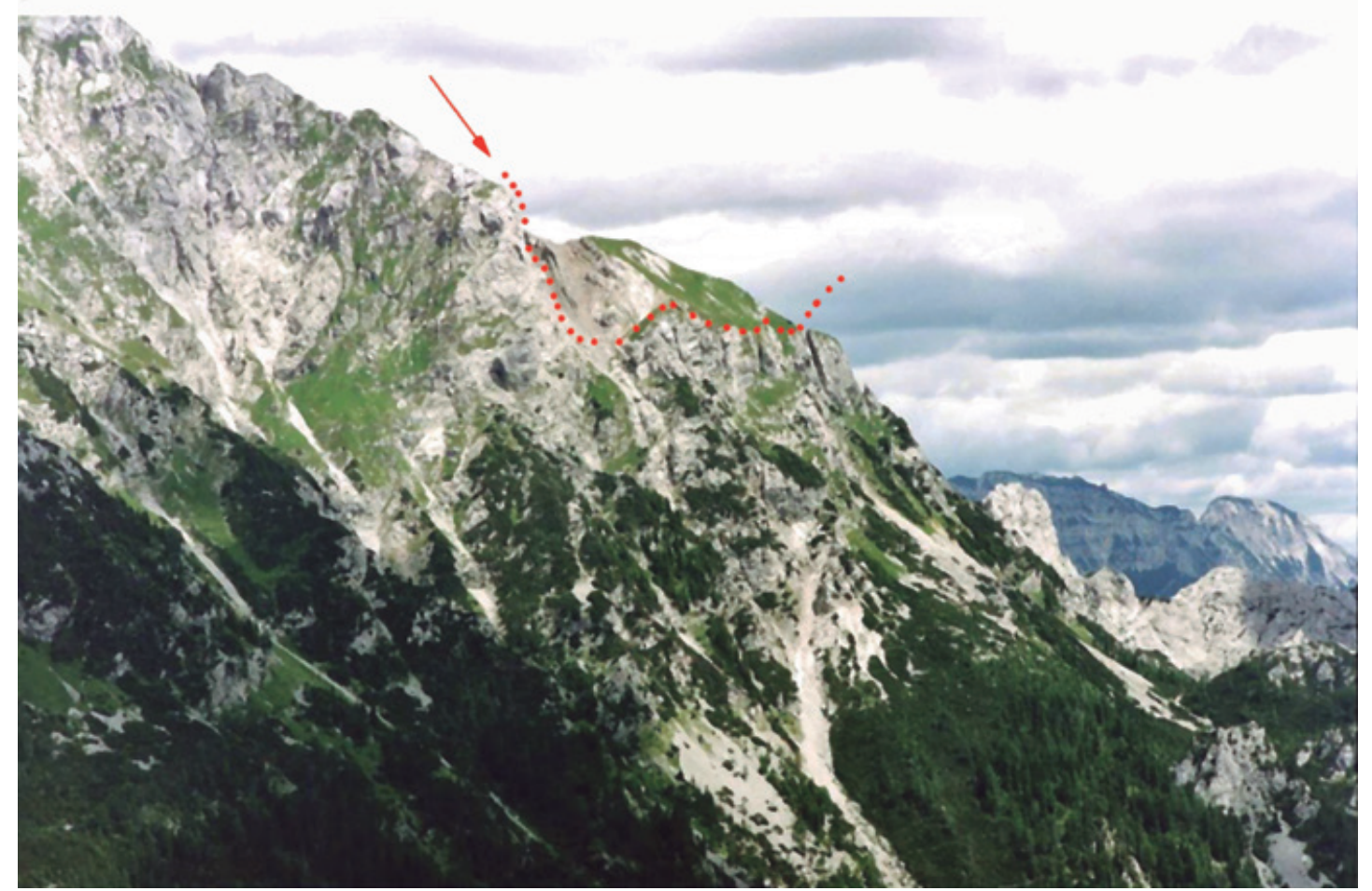

Figura 3. Vertiente sur del Rosskofel vista hacia el este. Véase, en medio de la cresta, la transgresión del Kasimoviense que corta de manera oblicua las calizas devónicas: es un karst volcado?

Figure 3. Southern slope of the Rosskofel; view to the east. Notice, in the central part of the crest, the transgression of the Kasimovian strata, which obliquely cut the Devonian limestone: is it a tripped karst?

caliza arrecifal karstificada del Devónico (Figura 3). En algunos casos, como por ejemplo, sobre la vertiente norte de la montaña $\left(46^{\circ} 33^{\prime} 28.43^{\prime \prime} \mathrm{N} 013^{\circ} 14^{\prime} 02.82^{\prime \prime} \mathrm{E}\right)$, se ven claramente los estratos transgresivos del Carbonífero superior que penetran en las cavidades del karst, envolviendo completamente éste último (Figuras 4 y 5 ).

En la vertiente meridional del mismo Monte Auernig, el corte, expuesto desde las estribaciones norte del macizo de Malvueric (Malurch), apéndice oriental del Rosskofel hasta la cima de Auernig, es muy instructivo. Se vislumbran en el fondo del Río Winckel, de sur a norte: las calizas devónicas solapándose hacia el norte. En este lugar, Heritsch (1936, Tb III perfil V) muestra una banda de "Nassfeldschichten" (Carbonífero superior) incrustada en el contacto. Más lejos, la vertiente norte del valle está constituida por formaciones de areniscas y esquistos oscuros con cantos rodados de lidita, areniscas verdosas con elementos de diabasa, más allá, areniscas gruesas verdosas con cantos rodados de cuarzo, cuarcita, lidita, a menudo, toscamente redondeados. Por encima, se distinguen "esquistos conglomératicos" (Heritsch 1936, p. 146) con cantos decimétricos de cuarcitas, frecuentemente del Silúrico inferior, y por encima esquistos grises o negros. Dichos estratos, según el autor citado, son del "Hochwipfelkarbon". Estos últimos suben prácticamente hasta la planicie del Passo di Pramollo (Nassfeldpass) y bajo la Casera Auernig (Figura 6).

Vienen por encima los estratos del Carbonífero superior, en dos partes distintas. El perfil fue detallado por Schellwien (1892), Frech (1894 p. 312), Geyer (1896, p. 159), o posteriormente, de entre una larga serie de científicos, por Pasini (1963), que estableció una bioestratigrafía detallada basada en la micropaleontología. Este último autor, como él mismo indica, tomó las muestras según dos cortes de terreno distintos, separados por las praderas pantanosas del paso de montaña de Nassfeld-Passo di Pramollo. No existe continuidad alguna de afloramiento, ni siquiera la posibilidad de observación, entre el final de su corte occidental (muestras $n^{\circ} 996$ a 1002) y la base de su corte más oriental (vertiente sur del Monte Auernig, muestras Pasini $n^{\circ} 1003$ a 1021). El primer grupo pertenece a un nivel "Moscoviense a Kasimoviense basal" y en particular con Pseudostaffella sp., Ozawainella cf. kumpani Rauzer-Chernousova, Protriticites pramollensis, Wedekindellina sp. y Fusiella sp. indet. El segundo grupo pertenece al Gzheliense superior, con Kahlerella alpina alpina (Schellwien), Quasifu- 


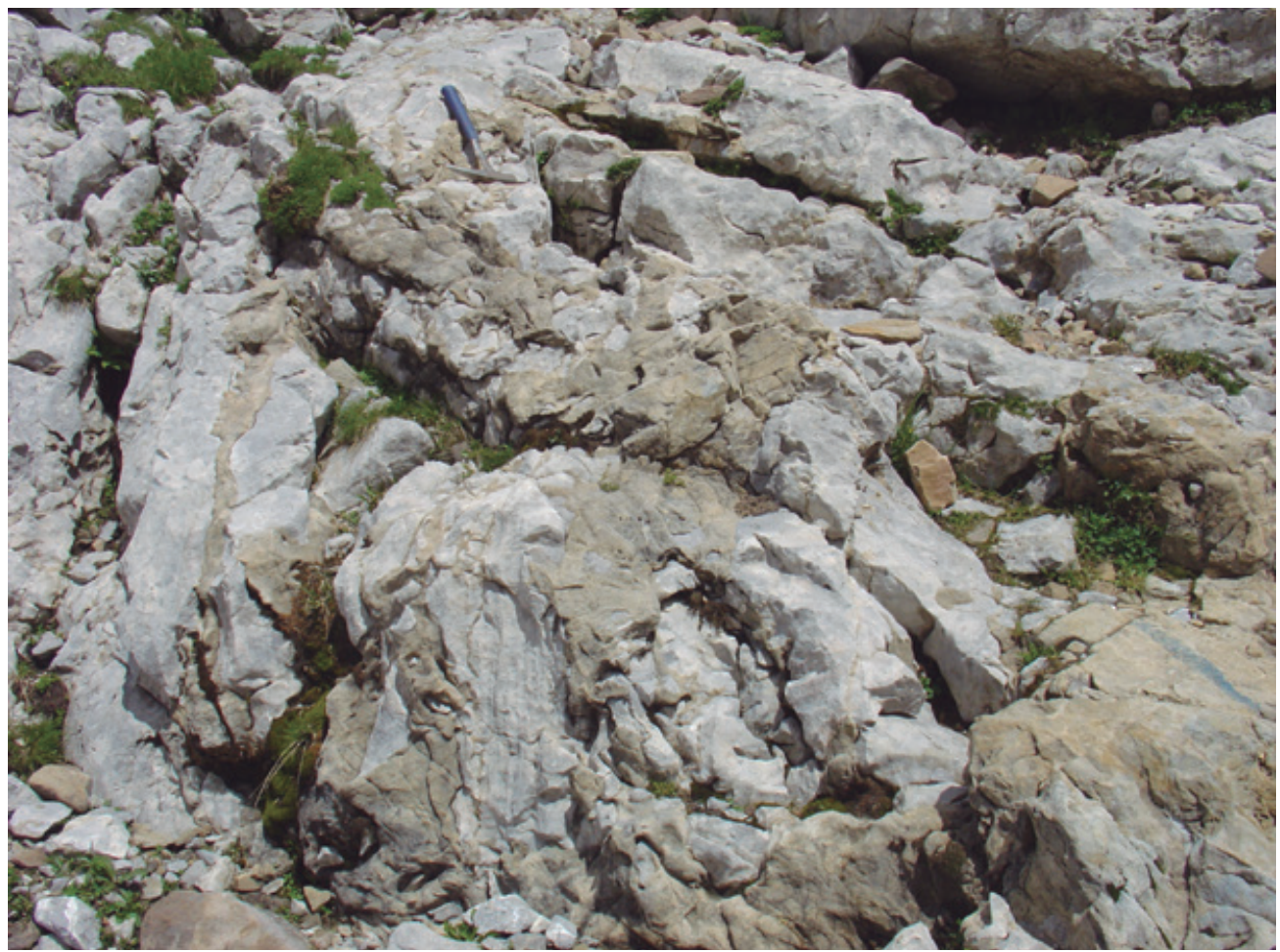

Figura 4. Vertiente norte del Rosskofel : la base de la transgresión del Carbonífero superior incrustada en el relieve kárstico de calizas devónicas.

Figure 4. Northern slope of the Rosskofel: the base of the transgression of the Upper Carboniferous is encrusted in the karstic relief of the Devonian limestone.

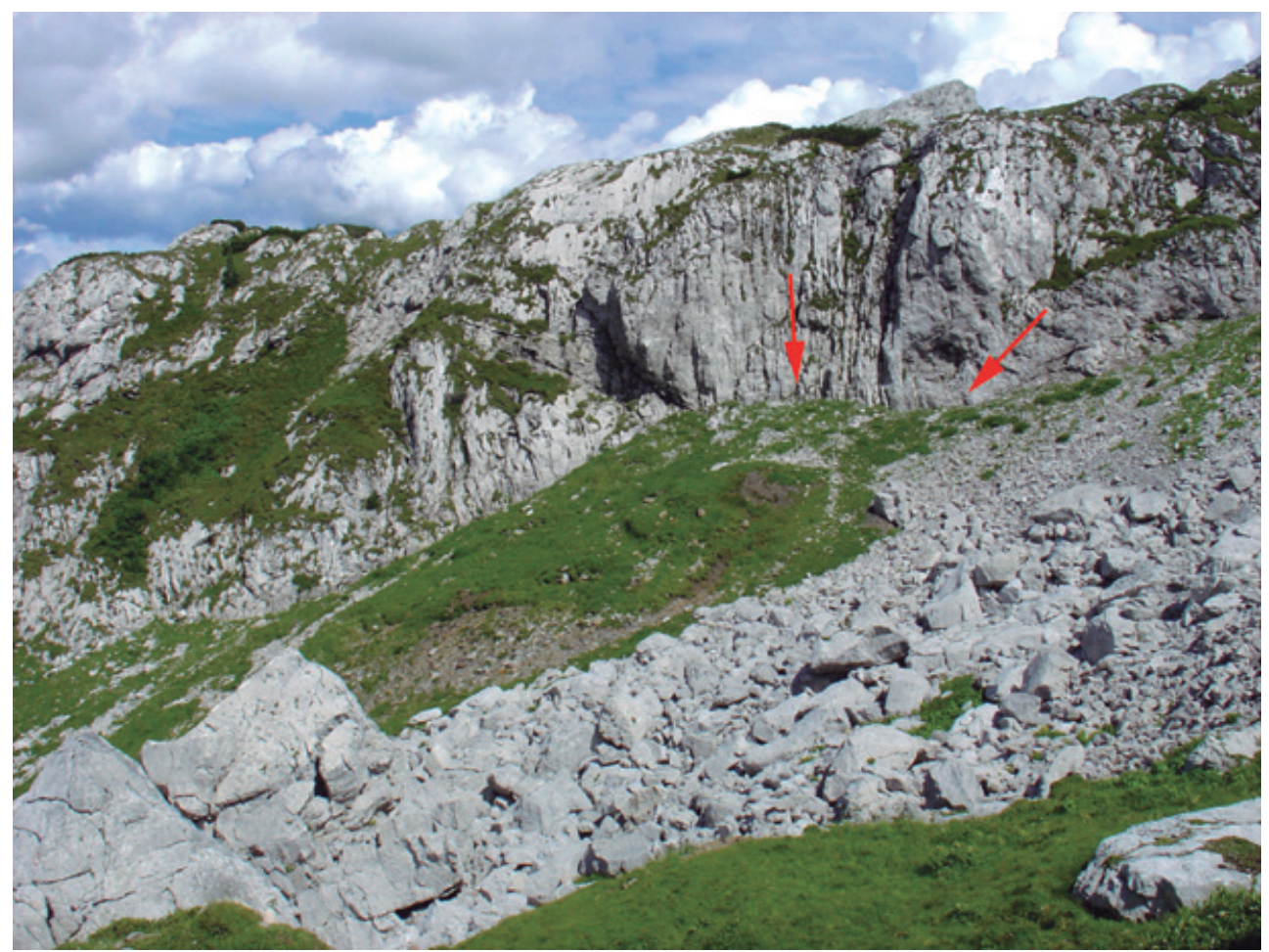

Figura 5. Vertiente norte del Rosskofel: Carbonífero superior transgresivo en una dolina de calizas devónicas.

Figure 5. Northern slope of the Rosskofel: Upper Carboniferous transgressive in a doline of the Devonian limestone. 


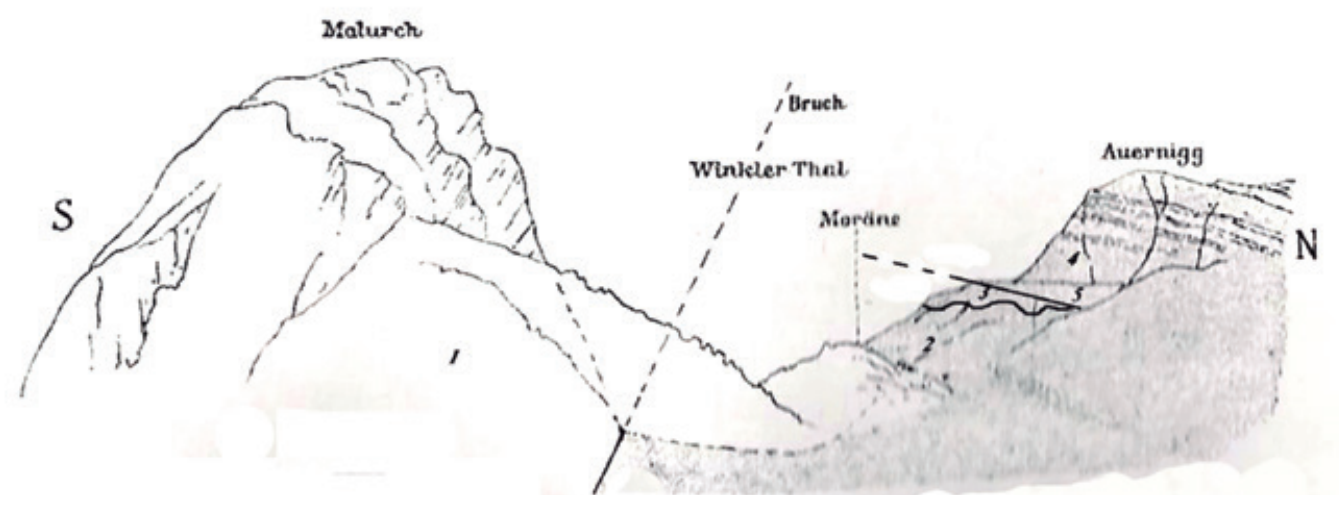

Figura 6. Sección desde el Monte Malvueric (Malurch) hacia el Monte Auernig. Según Suess (1892), modificado. 1 : Devónico del Rosskofel 2: Estratos de Hochwipfel 3: Kasimoviense del ámbito de Zollner See 4 : Gzheliense - Orenburgiense del ámbito de Auernig 5 : brecha tectónica.

Figure 6. Section from Mount Malvueric (Malurch) up to Mount Auernig; according to Suess (1892), modified. 1. Devonian of the Rosskofel. 2. Hochwipfel strata. 3 Kasimovian of the Zollner See realm. 4. Gzhelian-Orenburgian of the Auernig realm.

sulina longissima (Moeller), fragmentos de Triticites sp., etc. Esta serie va subiendo sin cambios de facies (muestras Pasini $n^{\circ} 1018$ a 1021) hasta niveles con fauna que son ya del Asseliense, con Staffella cf. moellerana Thompson, Nankinella sp. ind., Sphaerulina aff. crassispira Lee, "Pseudofusulina" sp., etc.

De hecho, los dos afloramientos están separados por un importante contacto tectónico que limita al sur el Monte Auernig. Así pues, para convencerse de eso, se pueden contar los estratos, bien individualizados, al norte y al sur de esta montaña. Sobre el terreno, se

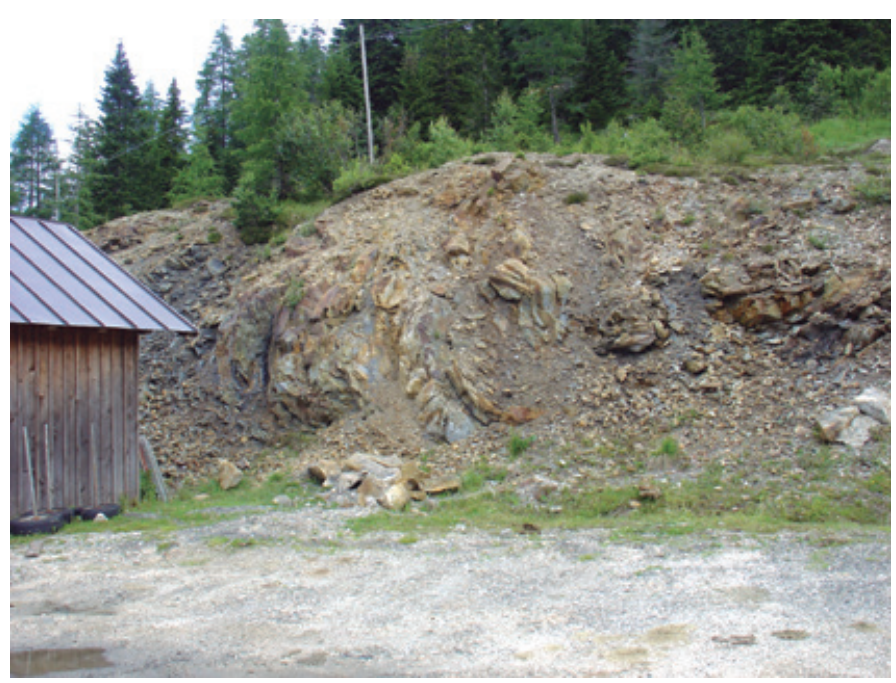

Figura 7. El contacto tectónico de base de los estratos gzhelienses del ámbito de Auernig en el punto $46^{\circ} 33^{\prime} 25^{\prime \prime} \mathrm{N}-013^{\circ} 16^{\prime} 44.88^{\prime \prime} \mathrm{E}$. Figure 7. Basal, tectonic contact of the Gzhelian strata of Auernig realm in the locality $46^{\circ} 33^{\prime} 25^{\prime \prime} N-013^{\circ} 16^{\prime} 44.88^{\prime \prime} E$. constata que el grupo de estratos kasimovienses ocupa un montículo en el lado oeste de la carretera Pontebba-Nassfeld (en el punto $46^{\circ} 33^{\prime} 21.13^{\prime \prime} \mathrm{N}-013^{\circ} 16^{\prime}$ $41.41^{\prime \prime}$ E). En cambio, más allá del área pantanosa, se puede seguir el accidente tectónico desde el albergue "Al Gallo Forcello" (del lado italiano de la frontera, $46^{\circ}$ $33^{\prime} 25^{\prime \prime} \mathrm{N}-013^{\circ} 16^{\prime} 44.88^{\prime \prime} \mathrm{E}$, Figura 7) y hacia la Casera Auernig. En la ladera tras ese contacto, inclinado hacia el norte, apuntan esquistos negros de Hochwipfel, mientras que en la parte que se halla por encima del contacto una brecha con elementos mayoritariamente plurimétricos a pluridecamétricos sostiene los estratos de edad gzheliense del Monte Auernig. Los elementos de esta brecha son esquistos negros de tipo Hochwipfel, dolomías blancas, brechas calcáreas o siliciclásticas y calizas grises ligeramente bioclásticas.

Las dolomías son dolosparitas segundarias en mosaico, sin granos identificables. La brecha contiene, sobre todo, elementos carbonatados. La parte más importante está constituida por "packstones" que contienen Parathuramminidae, concretamente, Suleimanovella paulis (Bykova); algunos son calizas nodulares con Globochaete ex gr. alpina Lombard, microfacies clásica del Givetiense y Frasniense de Europa suroccidental (Pirineos, Montaña Negra, etc.). Es la facies Ilamada comúnmente "griotte". Los elementos restantes son lutitas probablemente también de edad devónica.

Las calizas fosilíferas $\left(46^{\circ} 33^{\prime} 21.60^{\prime \prime} \mathrm{N}\right.$ $\left.013^{\circ} 16^{\prime} 52.78^{\prime \prime} \mathrm{E}\right)$ son (muestras AC 99-3 y AC 99-13), respectivamente, una caliza litoclástica y bioclástica con escasos Praeobsoletes sp. De forma detallada, la 
caliza AC 99-13, rica en microfauna, contiene la asociación siguiente: escasos crinoideos y fragmentos de bivalvos, escasas algas incertae sedis Komia; pequeños foraminíferos tales como Diplosphaerina inaequalis (Derville), Spireitlina conspecta (Reitlinger), Endothyra sp., Iriclinella sp., Bradyina cf. samarica Reitlinger, $B$. magna Roth y Skinner, Climacammina bosbiensis Bogush, Tetrataxis cf. parviconica Lee y Chen, Polytaxis planispiralis (Reitlinger), Globivalvulina mosquensis Reitlinger, Calcitornella sp., Syzrania cf. confusa Reitlinger; y fusulinidos: Praeobsoletes aff. tethydis (Igo), $P$. aff. burkemensis (Volozhanina) y P. sp. 3.

En realidad, esta serie es la continuación de la que se encontró más al oeste, con la particularidad de la ausencia local del Pérmico superior transgresivo (Gröden y Bellerophon), probablemente como consecuencia de una laminación tectónica. Esta interpretación viene confirmada por el hecho que, del otro lado (norte) del macizo de Gartnerkofel, el conjunto Carbonífero terminal a Ladiniense (ámbito de Auernig y su cobertura triásica) está separado de su sustrato por un conjunto de escamas de las cuales dos están constituidas por Gröden directamente transgresivo sobre el Carbonífero superior (cf. Heritsch, 1936 p. 144, 145). Un pequeño indicio de la existencia en profundidad de yeso del Pérmico superior se confirma por una fuente fuertemente sulfurada (Schwefelquelle) en el camino de Nassfeld a Watschiger Alm (al norte del Monte Auernig).

\section{El ámbito de Auernig (Figura 8)}

Presenta varias diferencias respecto al ámbito precedente:

- Su base siempre está en contacto tectónico con su sustrato (Figura 7), y sus raíces son desconocidas (Figuras 9 y 10).

- La serie comienza, según en qué lugares, en diferentes niveles del Gzheliense.

- Se ve afectado por erosiones y lagunas relacionadas con la fase saaliense, pero dichas lagunas son de poca amplitud, sin relación con el Pérmico del dominio descrito anteriormente.

- Los niveles rojos post-saalienses transgresivos son muy diferentes de los del Gröden clásico.

- El Pérmico superior se encuentra representado por calizas y dolomías con Bellerophon, pero de una facies muy diferente y mucho más netamente marina que las de misma edad del ámbito precedente, así como del conjunto de los Alpes meridionales limítrofes.

- Está totalmente desprovisto de esquistosidad, mientras que el ámbito de Zollner See se halla afectado por una esquistosidad de dirección $110^{\circ}$.

\section{Estratigrafía}

La descripción estratigráfica y bioestratigráfica de las series del ámbito de Auernig ha sido objeto de un volumen impresionante de análisis y publicaciones, cada vez más precisas. Puede que en el futuro el ritmo de dichos análisis sea, a minima, el mismo.

La serie estratigráfica - reconstituida - comprende un número importante de "formaciones" definidas por varios autores, sobre todo desde finales del siglo XX. Para no menguar la claridad de lo expuesto, nos limitaremos a la división estratigráfica clásica, tal como fue definida por Geyer (1895) y precisada sucesivamente más tarde (Heritsch et al. 1934; Heritsch 1936; Kahler 1983, 1985, 1986; Kahler y Krainer 1993; Venturini 1990a,b; Cassinis et al. 1998; Vachard y Krainer, 2001a,b; lista no exhaustiva). En todo momento despegada en su base, la serie comienza en un Gzheliense formado por alternancias de calizas marinas con fusulínidos y bancos detríticos con restos de plantas. Por encima, la parte inferior del Pérmico inferior está compuesto de tres términos, dos calizas que se enmarcan en el término "Grenzlandbänke" que es un elemento recurrente de las facies gzhelienses subyacentes; por encima, la parte superior del Pérmico inferior se halla representada por la "caliza de Trogkofel" erosionada por una brecha; la formación detrítica roja llamada "de Gröden" transgrede por encima, dominada a su vez por la formación carbonatada con Bellerophon que se convierte en su techo en el Scytiense subalpino. La descripción estratigráfica y bioestratigráfica se encuentra en remarcables publicaciones de Forke et al. (2006), Davydov et al. (2013) y Krainer et al. $(2019 a, b)$.

\section{Los afloramientos orientales}

En los Alpes Cárnicos no se observa ningún corte continuo del conjunto de toda la serie. La sección más representativa - y conocida con mayor anterioridad del Carbonífero superior es el mismo Monte Auernig, a partir del accidente de la Casera Auernig y hacia arriba hasta el Monte Corona (Krone, $1832 \mathrm{~m}$ ). La base es un contacto tectónico; el conjunto está compuesto por una alternancia de conglomerados, esquistos y areniscas con plantas, calizas con fusulínidos, de edad gzheliense, llegando a una caliza con fauna que ya es del Asseliense (Pasini 1963). Hacia la parte alta de la serie estratigráfica, la sección más completa se localiza en la vertiente Reppwand-Kühweger Köpfl bajo el flanco NO del macizo triásico de Gartnerkofel (Figura 8). Hay que destacar que un accidente tectónico importante y conocido con mucha anterioridad, que transcurre al sur de Gartnerkofel lo separa de la serie gzheliense de la región de Auernig. 


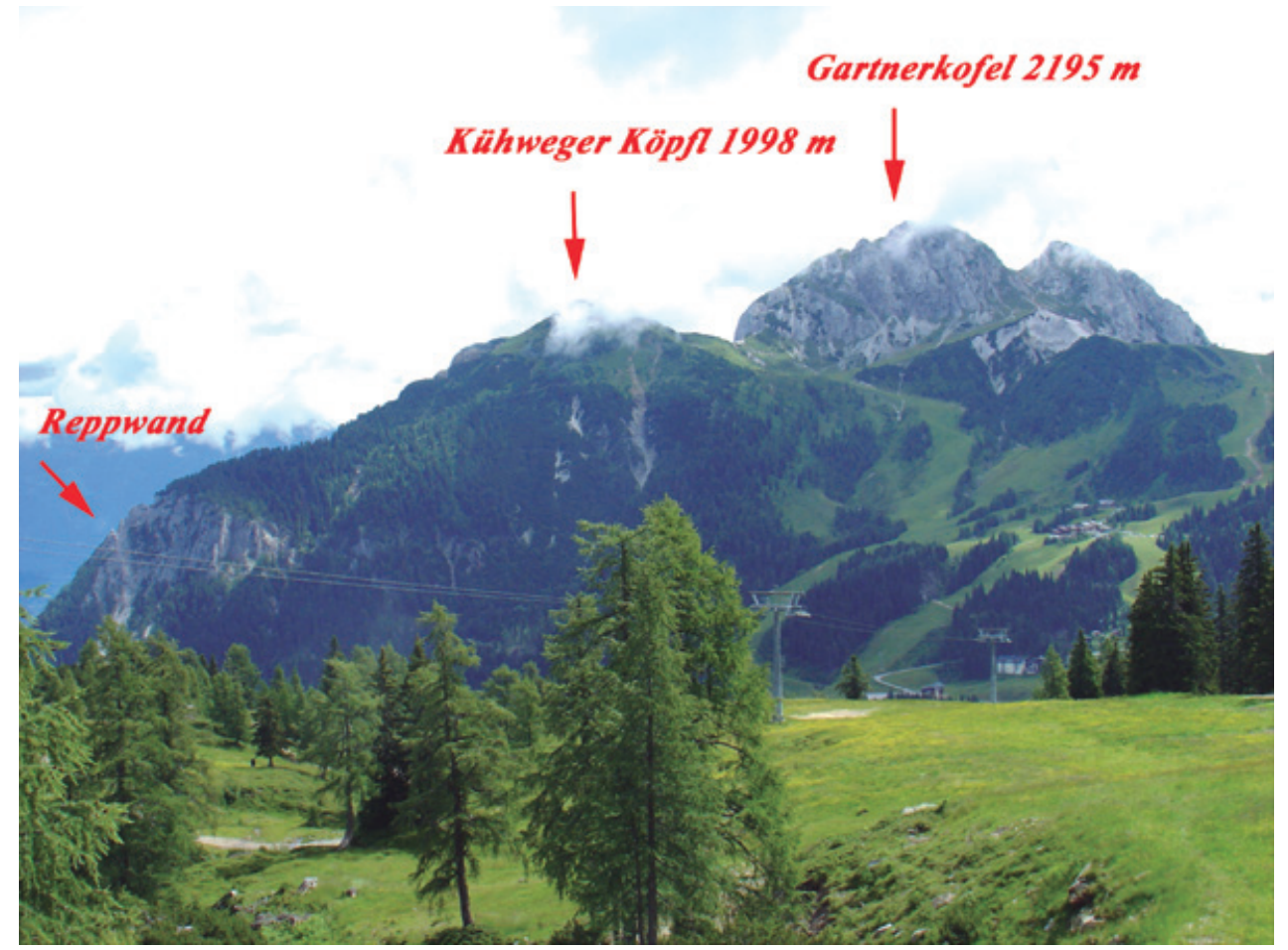

Figura 8. Reppwand, Kühweger Köpfl y Gartnerkofel. Vista hacia el NNE.

Figure 8. Reppwand, Kühweger Köpfl and Gartnerkofel. View to the NNE.

- La base de esta sección es imprecisa, ya que está fuertemente tectonizada, formada por varias escamas (entre dos y cuatro siguiendo las secciones al norte de Gartnerkofel, de Oselitzen a Nassfeld, y en Oselitzen - desde la cota 1120 hasta el punto con cota 1320 - así que, de abajo a arriba, por debajo de Schwarzwipfel (cf. Frech 1894; Geyer 1896; Heritsch 1936; etc.). De esas escamas, que se alzan sobre los mármoles de "Ederdecke" y arrastran jirones de la formación de Hochwipfel, algunas incluyen estratos gzhelienses mezclados tectónicamente con jirones de caliza de Trogkofel, y otras, estratos kasimovienses, dominados en transgresión por el Gröden y el Bellerophon (Schwarzwipfel).

- La parte del Pérmico inferior ("Estratos de Rattendorf" de Heritsch et al. 1933), comprende sus tres términos típicos: La "caliza inferior con Pseudoschwagerinas" tiene allí un espesor de cerca de 60 metros, aunque su espesor primario debió ser mayor, dado que su base está truncada siguiendo una superficie de despegue por encima de los estratos gzhelienses. Se trata de una caliza masiva a medianamente estratificada en zonas, de color claro. Esta caliza es una biomicrita con un porcentaje irregular de microorganismos en su interior, que va aumentando hacia la parte alta. Se distinguen pequeños gasterópodos y braquiópodos, crinoideos y algas como Eugonophy-
Ilum y Anthracoporella. En ocasiones, se encuentran vetas biosparíticas, con frecuencia dolomitizadas, con fragmentos de equinodermos. El fósil característico de este nivel, Pseudoschwagerina alpina Kahler, sólo aparece en la mitad superior de la caliza. Por encima, se encuentra un afloramiento minúsculo de la facies más detrítica de los "Grenzlandbänke". Se sitúa al pie de Reppwand, en medio de la formación de la pendiente, sin que se pueda afirmar con certeza el carácter normal de su yacimiento, teniendo en cuenta la fuerte tectónica en dicho lugar. La "caliza superior con Pseudoschwagerinas" aparece en diversos lugares a través de formaciones recientes. Sin embargo, sólo se puede observar en continuidad estratigráfica con la caliza de Trogkofel situada por encima, en el extremo norte del acantilado de Reppwand. El acantilado está constituido por calizas bien veteadas de color rojizo sucio, con interestratos margosos rojos o verdes. Este último hecho fue puesto de manifiesto por Kahler and Prey (1963).

- La parte superior del Pérmico inferior ("caliza de Trogkofel"). Constituye la parte principal del acantilado y presenta aquí más de 200 metros de espesor media. Se trata de caliza arrecifal maciza que muestra, en esta sección, una dolomitización secundaria bastante significativa. Por esta razón, se examinó más detenidamente en el locus typicus (macizo deTrogkofel). En este 
corte de Reppwand, la cima de caliza de Trogkofel está erosionada y se observa, en ciertos lugares, una brecha o más bien, una pudinga monogénica, cuyos elementos calcáreos, centimétricos a decimétricos, provienen de la caliza subyacente. El espesor de esta formación no supera aquí una decena de metros ya que fue visiblemente erosionada antes de que se depositaron los estratos de Gröden que la cubren y puede hallarse localmente ausente. Su cemento puede ser silíceo muy fino, de color rojizo, o bien carbonatado, de color gris claro o beige. Esta brecha se asemeja a la brecha denominada "de Tarvis" por las razones que desarrollaremos más adelante.

- Estratos "de Gröden". Estos reposan sobre una superficie de erosión que afecta la brecha y puede alcanzar la caliza de Trogkofel. Se trata de pelitas que contienen escamillas muy finas de mica, esquistos arcillosos y margas foliadas, el conjunto de color rojo vivo o rojo teja. Así mismo, pequeños lentejones y nódulos de caliza de color amarillo sucio o gris y de marga dolomítica se encuentran intercalados. En ocasiones, se observan elementos de esas mismas formaciones que forman parte de microbrechas con estratos finos en los esquistos y pelitas. El espesor total de la formación en este lugar no sobrepasa los 40 metros. Hacia el techo, el color se vuelve más oscuro, pardo o violáceo, con niveles centimétricos de carniola parda. En todo el espesor de la formación, el color rojo puede dejar paso episódicamente a una coloración verde, amarillo sucio o incluso blanca; los niveles pelíticos más groseros pueden mostrar estratificaciones cruzadas, pero el predominio de la fracción fina y de los esquistos resulta muy marcada y característica. No se conocen fósiles en esta formación; fue descrita por primera vez por Geyer (1895) y estudiada a partir de entonces por numerosos autores (Heritsch, Kahler, Metz, etc.; ver lista bibliográfica). Dichos investigadores la han descrito como el equivalente de la formación de Gröden definida en el Tirol meridional (Richthofen 1860), datada en el Pérmico superior.

Esta datación se basa esencialmente en la posición estratigráfica, comprendida en este corte de Reppwand entre la caliza de Trogkofel - y la brecha - en la parte baja, y los estratos con Bellerophon del Pérmico terminal. Cabe subrayar, de entrada, que este tipo de "estratos de Gröden" es particular y se limita estrictamente al ámbito de Auernig. Se distingue de la formación de Gröden propiamente dicha por su escaso espesor ( $40 \mathrm{~m}$ frente 200 a $600 \mathrm{~m}$ ), la ausencia de detrítico grueso, especialmente de conglomerados con guijarros de cuarzo y elementos volcánicos, el alto porcentaje de la fracción arcillosa, la mejor cristalización de la illita (Buggisch et al. 1976) y la importancia de la bioturbación.
- Dolomias de la formación con Bellerophon. Sobre la formación precedente se apoya una dolomía oscura, bituminosa, con estratos finos de alrededor de 10 $\mathrm{m}$ de espesor; el pasaje es progresivo y continuo. Por encima de ese primer nivel se presentan unos $30 \mathrm{~m}$ de esquistos arcillosos verdes y violáceos con intercalaciones frecuentes de carniolas y dolomías de color amarillo sucio. Dichos estratos pasan progresivamente a una dolomía gris o gris amarillenta, a menudo bituminosa, cuyo espesor es de unos $200 \mathrm{~m}$. Las primeras decenas de metros están marcadas todavía por facies evaporíticas (carniolas), pero sin yeso. Hacia la base de la dolomía gris aparecen algas dasycladáceas y foraminíferos (Miliolata). Un poco más arriba, se distinguen ostrácodos y radiolarios en una roca esparítica, microesparítica y micrítica. Hacia el techo de la formación, se encuentran conodontos (Buggisch et al. 1976). Cabe constatar que los caracteres de dichos estratos permiten distinguir esta dolomía de Reppwand de la formación con Bellerophon típica, tal como fue definida en los Alpes Meridionales: la facies netamente marina predomina aquí. Se observan tránsitos importantes hacia una facies de mar abierta, con las dolomías estratificadas con foraminíferos y algas, radiolarios, etc. Las evaporitas presentan poca importancia, con ausencia de yeso, mientras que éste último está bien presente en el Pérmico terminal de los Dolomitas, así como de los Alpes Julianos, al sur de Gartnerkofel (Bagni di Lusnizza). Incluso la facies "Badiota", la más marina de las dos facies de los Alpes Meridionales, difiere de la de Reppwand por las alternancias de yeso y calizas de mar abierto. Esta última, como la formación que la precede, está bien delimitada en el dominio de Auernig. Bajo este punto de vista, dicho dominio se acerca más al del Save, en Eslovenia (estratos de Saschar) y del Velebit, en los Dinárides.

- Scytiense. Sobre la cara norte de la pequeña cima Kühweger Köpfl (1998 m) y a partir de una altitud de unos 1900 m, el corte de Reppwand muestra dolomías areniscosas grises, amarillo sucio o incluso rosadas, que se alternan con bancos margosos grises y parduzcos. En éstas, se pueden encontrar niveles oolíticos rosas, cuyos núcleos se componen con frecuencia de restos de gasterópodos ("Gastropodenoolith"). Ciertos niveles de calizas gris azulada contienen en ocasiones horizontes lumaquélicos, sobre todo de Claraia. Más arriba, se pasa progresivamente a una facies más detrítica en la que predominan los tonos rojizos, que se trata de esquistos arcillosos y de pelitas rojas muy finas, alternándose éstos con niveles oolíticos con alteración a menudo a tonos más claros, seguidos de dolomías areniscosas con estratos muy finos de color violáceo, rojo o verde, de arcillas y lutitas rojas, con frecuencia micáceas, así como de pelitas del mismo 
color. El espesor de este Scytiense es ligeramente superior a 70 metros. Los autores austríacos (cf. Kahler y Prey 1963) se inclinan fácilmente por la apelación de facies "Seis" en la parte baja de la formación y de facies "Campil" en la parte superior. No obstante, la base de la formación difiere de la facies Seis de los Alpes Meridionales, sobre todo por su mayor riqueza en carbonatos así como por la presencia, mucho más discreta en este caso que en Los Dolomitas (Servino!) de la fracción detrítica. Cabe subrayar, finalmente, la notable continuidad que caracteriza aquí el tránsito Pérmico-Triásico, hasta el punto que es imposible trazar el límite entre los dos niveles con precisión.

\section{Los afloramientos occidentales: Tressdorfer Höhe,} Trogkofel, Zweikofel, Schulterkofel (Creta di Lanza)

El extremo oeste del área de afloramiento del ámbito de Auernig se caracteriza por la existencia de varios afloramientos. Ahora bien, debido a la intensa tectonización, es imposible describir en ella una sección continua y coherente en el campo, y la sucesión está reconstituida a partir de diferentes afloramientos y tramos de sección. Tressdorfer Höhe es una pequeña cima calcárea al Oeste-Noroeste de Nassfeld y con cota $1875 \mathrm{~m}$. Pertenece (Heritsch 1928, 1936) en la cara norte de un anticlinal fallado de núcleo gzheliense y se compone de la "caliza inferior con Pseudoschwagerinas". El interés de dicho afloramiento estriba en que presenta una serie carbonatada pero también detrítica ordenada en ciclos, que empiezan con una sedimentación detrítica y evolucionan cada uno de ellos hacia la parte alta de los sedimentos con predominio carbonatado. Buggisch et al. (1976) distinguen en total cuatro ciclos en los Alpes Cárnicos. La parte detrítica basal de los ciclos está formada por lutitas, arcillas y areniscas - con algunas calizas intercaladas- mientras que la sedimentación carbonatada va aumentando hacia la parte alta del ciclo hasta invadir el conjunto. En esta fracción carbonatada predominan las micritas (biomicritas) con algas y foraminíferos. La parte superior de ese jirón de Tressdorfer Höhe contiene el fósil característico de dicho nivel, Pseudoschwagerina alpina Kahler.

El grupo de Trogkofel-Zweikofel es un conjunto en el que afloran, en macizos más o menos desgarrados tectónicamente, todos los términos conocidos del Pérmico inferior de los Alpes Cárnicos. Este grupo está limitado al SSE por el conjunto de accidentes de Rudnig Sattel que lo separan del área de afloramiento de los estratos gzhelienses, tectónicamente superpuestos, a su vez, al dominio de Zollner See. En dirección hacia los contrafuertes norte del macizo de Rosskofel hacia el de Trogkofel por el puerto de montaña de Rudnig
Sattel, se atraviesa primero un conjunto de accidentes tectónicos, luego se entra en una formación llamada por Kahler (1932) "Grenzlandbänke". Se trata de una formación esencialmente detrítica, pero que contiene bancos calcáreos más o menos lenticulares. Tiene un gran parecido con los estratos gzhelienses de Auernig $y$, de hecho, se ha hablado de "recurrencia de la facies Auernig" (Kahler y Prey 1963). La parte detrítica está constituida por areniscas, lutitas, pelitas y conglomerados. Los elementos de éstos últimos son esencialmente guijarros de cuarzo blanco y escasos guijarros de lidita. Los bancos calcáreos están compuestos de caliza gris finamente estratificada, micrítica a biomicrítica, rica en algas y grandes fusulínidos. Este tramo se caracteriza también por la presencia frecuente de oncoides, a menudo de gran talla (centimétricos a pluricentimétricos) que rodean núcleos, y son formados por una cyanobacteria (Girvanella). Los fusulínidos característicos se encuentran en las calizas, así como en las lutitas esquistosas. Kahler (1934) y Kahler y Kahler (1937) identificaron: Pseudoschwagerina confinii, P. turbida, P. carniolica, P. aequalis y P. extensa. Los numerosos braquiópodos permitieron a Heritsch (1933) establecer una primera estratigrafía, que fue completada más tarde por corales tales como Rossophyllum densiseptatum Heritsch, Siphonophyllia sophiae Heritsch etc. Usando los fusulínidos, Kahler (1973) atribuyó este nivel al Asseliense. Un hecho interesante respecto a la formación Grenzlandbänke es la aparición sistemática de coloraciones rojizas en el sedimento, siendo la primera aparición de este tipo de coloración en los sedimentos post-hercinianos en la región, y la que va a marcar los sedimentos detríticos permo-scytienses. En este grupo son sobre todo las areniscas las que toman un color rojo vivo a teja. En el NE de Rudnig Sattel, el pequeño macizo con cota en 2004 metros en los antiguos mapas austríacos, rodeado de Grenzlandbänke, se halla rodeado en su base de "caliza inferior con Pseudoschwagerinas" que pasa, hacia la parte alta, a caliza de Trogkofel. Su contacto, en la base se encuentra mecánicamente modificado, de forma en este caso que no se puede hablar de contacto estratigráfico normal. Esta base está constituida por aportes detríticos bastante groseros - areniscas y conglomerados con guijarros de cuarzo - estrictamente limitados en la parte basal. Por encima se localiza una caliza gris claro, finamente estratificada, que pasa más arriba a una caliza roja, y progresivamente hacia arriba a la caliza de Trogkofel. Se trata de una caliza micrítica y esparítica, de la que varios niveles presentan abundantes oncoides generados alrededor de algas filoides. En ella, la fauna y la flora son ricas y diversificadas, las algas dasycladáceas son muy abundantes (Mizzia, Epimastopora, Pseudoepimastopora) 
así como Neoanchicodium en las facies micríticas (Buggisch et al. 1976; Krainer et al. 2019a). Se pueden encontrar también en ella muchos otros grupos de organismos (gasterópodos, equinodermos, tubiphytidos, briozoários, etc.). Ciertos niveles registran muchos foraminíferos palaeotextuláriidos, y fusulínidos, que, en ocasiones, forman verdaderas lumaquelas. Las principales especies son (Kahler 1974) Zellia heritschi Kahler y Pseudoschwagerina pulchra, que datan este nivel del Hermagoriense (Davydov et al. 2013).

Hacia el oeste se encuentra la caliza de Trogkofel (Creta di Aip, 2279 m) que constituye 400 metros de pared vertical y le confiere un rasgo característico al paisaje. Dicho macizo está totalmente constituido de caliza del mismo nombre. El sendero que llega de Rudnig Sattel, y lo aborda en su ángulo SE, permite ver que la caliza reposa directamente sobre los "Grenzlandbänke", tras la desaparición por laminación de la "caliza superior con Pseudoschwagerinas" que se encuentra, en cambio, un poco más al oeste y que se puede seguir a lo largo de la base de la pared meridional. El contacto basal del macizo se halla afectado tectónicamente, hecho ya indicado por Heritsch (1928). En este ángulo SE, la caliza de Trogkofel está brechificada; sin embargo, se observan granos de cuarzo detríticos bien redondeados, de una talla media de $1 \mathrm{~cm}$. Este aporte detrítico fue indicado inicialmente por Schwinner (1927), quién menciona igualmente la presencia de granos de lidita. La caliza en sí misma se halla mal estratificada en su base y se vuelve pronto completamente masiva; es de color claro, blanca, gris claro o rosa, con fractura del mismo color. En ciertos lugares puede pasar a niveles de brecha intraformacional con frecuencia dolomitizada, aunque su aspecto más característico macroscópicamente es el color rojo vivo, violeta o rojo teja, que la afecta por manchas irregulares. Esta pigmentación se debe a impregnaciones de una materia calco-síltica, que contiene, en ocasiones, escamillas muy finas de filitas, que rellenan cavidades, grietas o fracturas de la caliza masiva. Se piensa en un relleno tardío de las diversas cavidades, post-litificación de la caliza, tras un hiato de sedimentación y, probablemente, una fase de emersión. Esta idea viene reforzada por el hecho de que el mismo tipo de materia entra en la composición de la materia detrítica fina de la brecha de Tarvis de la meseta somital. En lámina delgada, la caliza deTrogkofel se presenta como un carbonato biomicrítico, en el que se encuentra frecuentemente una facies con numerosas dasycladáceas o con Tubiphytes Maslov. La fauna de dicha caliza es muy abundante, aunque, desgraciadamente, una dolomitización secundaria hace que los microfósiles sean a menudo indeterminables. Este es el caso del extremo oeste de la pared meridional en la que las Pseudoschwagerinas forman verdaderas acumulaciones.

En la meseta somital de Trogkofel se halla una pudinga con cantos calcáreos o dolomíticos de talla decimétrica. El cemento es carbonatado de color gris o finamente areniscoso de color rojo teja; para Heritsch (1936) se trata de una brecha arrecifal intraformacional, pero en lo que nos concierne, esta brecha reposa sobre la caliza, como ya lo pensaba Geyer (1896), erosionándola muy intensamente. Se trata de la brecha de Tarvis localizada en la parte meridional de la meseta, donde el espesor de la caliza alcanza como máximo $200 \mathrm{~m}$, y que está ausente en su parte septentrional, en la que la caliza de Trogkofel alcanza los 400 $\mathrm{m}$. Los elementos de la brecha provienen exclusivamente de la caliza subyacente, y su espesor visible tras la erosión varía de 10 a $20 \mathrm{~m}$.

El nivel pérmico más elevado que localizamos en este grupo occidental de afloramientos del ámbito de Auernig está representado por un pequeño afloramiento de areniscas rojas de Gröden que se encuentra en condiciones tectónicas complejas en la cima de Troghöhe, pequeño macizo en el este deTrogkofel.

La bioestratigrafía de la caliza de Trogkofel precisa algunas observaciones y aclaraciones: en el locus typicus la caliza fue datada como Sakmariense oYakhtashiense (Krainer et al. 2019a,b). Desgraciadamente, el término "estratos deTrogkofel" ha sido utilizado en otros lugares (Eslovenia, Dinárides centrales...) en los que series calcáreas pero también detríticas suben hasta el Artinskiense e incluso, en algunas zonas, más jóvenes. Sobre esta cuestión de edad véase también Krainer et al. $(2019 a, b)$. Más cerca de la región a la que nos referimos, los afloramientos de Col Mezzodi, cerca de Forni Avoltri, estudiados por primera vez por Gortani (1906) muestran un Carbonífero superior con material detrítico, calizas pérmicas, una serie areniscosa gruesa de tipo Gröden y una serie evaporítica con Bellerophon. No obstante, en cuanto a la edad de las calizas, cabe indicar que, según Buggisch et al. (1976) "Ias calizas de Trogkofel" podrían subir mucho más que las de la localidad tipo; porque "alcanzan el dominio con Neoschwagerinas" ("Bereich mit Neoschwagerinen", p. 151). Se trataría entonces del Pérmico medio. Ahora bien, tras examinar las faunas descritas por Gortani (1906), por un lado, y el estudio de las nuevas secciones por el otro (Argyriadis 1978), cabe constatar que las calizas, bajo la transgresión con erosión por la brecha de base de las areniscas de Gröden, deben pertenecer exclusivamente en el Pérmico inferior

En lo que respecta a la serie de Col Mezzodi, cercana a Forni Avoltri, pudiera muy posiblemente pertenecer al ámbito de Zollner See. 


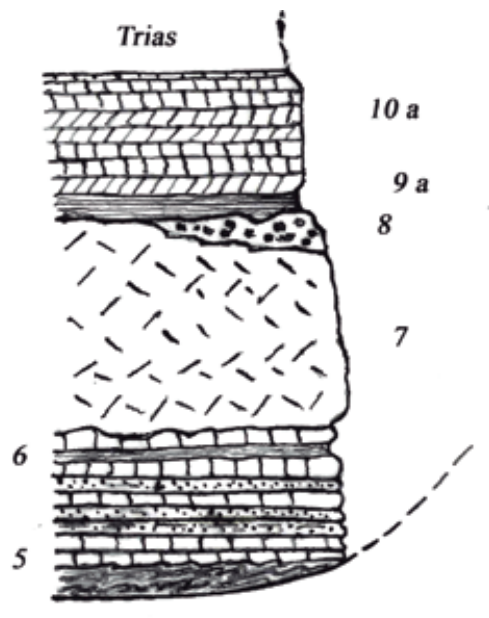

AUERNIG

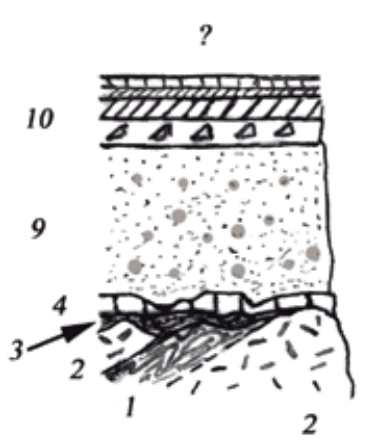

ZOLLNER SEE

Figura 9. Columnas estratigráficas sintéticas de los dos dominios definidos. 1 : Silúrico 2 : Devónico arrecifal 3 : Hochwipfel $4:$ Kasimoviense 5: Gzheliense- Orenburgiense de Auernig 6: Pérmico inferior ("calizas con Pseudoschwagerina") $7:$ Calizas deTrogkofel $8:$ Brecha deTarvisio 9: Estratos de Gröden 9a: Esquistos rojos "de Gröden" 10 : "Estratos de Bellerophon" con evaporitas (facies "Fiamazza" de los Alpes Meridionales) 10a: "Estratos de Bellerophon" dolomias de facies netamente marina.

Figure 9. Synthetic, stratigraphical columns of the two defined realms. 1: Silurian; 2: Reefal Devonian; 3: Hochwipfel; 4: Kasimovian; 5: Gzhelian-Orenburgian from Auernig; 6: Early Permian ("limestone with Pseudoschwagerina"); 7: Trogkofel Limestone; 8: Tarvis Breccia; 9: Gröden strata; 9a: Red shales "from Gröden"; 10: "Bellerophon strata" with evaporites (facies "Fiamazza" of the Southern Alps); 10a: "Bellerophon strata" represented by dolomites displaying a marked marine facies.

\section{Extensiones y correlaciones}

En base a los datos antes expuestos, es interesante investigar, sobre la periferia mesogeana (es decir tethysiana) del edificio hercínico, las trazas de un ámbito en el que el Carbonífero marino alcanzaría la altura suficiente para ser transgredido a continuación por un Pérmico rojo, él mismo seguido por un Pérmico terminal carbonatado y marino y luego, por un Triásico de las mismas características.

\section{Extensión hacia el este}

Hacia el este y el sudeste se extiende una amplia región (Karawanken, Alpes Julianos, Alpes Dináricos así como la parte meridional de los Cárpatos y cuenca Panónica), que no se puede integrar en este trabajo por razones de espacio disponible. Sin embargo, hay que señalar que los dos tipos de ámbitos pueden encontrarse, con un predominio cada vez más acusado del ámbito de Auernig, ya que la Mesogea pérmica se extiende progresivamente hacia el sudeste actual. No obstante, se puede reconocer el ámbito de Zollner See en el paraautóctono de la montaña de Bükk que emerge de los sedimentos recientes de la cuenca Panónica: un Carbonífero superior que alcanza al Gzheliense se halla recubierto por areniscas rojas pérmicas dominadas a su vez por calizas datadas por conodontos del Pérmico terminal, y por encima por el Tríasico basal de facies Seis (cf. Balogh 1964, Szalai 1979 y Haas et al. 1999). El mismo tipo de ámbito paleogeográfico se puede reconocer en Serbia occidental, en la isla de Chíos (Grecia), en la serie de Gökdag en Anatolia, etc. Argyriadis (1978) ofrece une relación más exhaustiva.

\section{Extensión hacia el Oeste}

Hacia el oeste, es decir hacia la región en la que el edificio varisco está bien establecido y generalizado. El ámbito de Auernig ya no aparece en esta dirección. Aparentemente, los Alpes Cárnicos han sido la parte distal afectada por los grandes mantos de corrimientos que lo trasportaron desde las regiones dináricas (Termier 1922). Solamente hacia el suroeste pueden persistir probables trazas de ese tipo de ámbito: en la Toscana meridional, por ejemplo, en formaciones anquimetamórficas, Pasini (1991) señala fusulínidos del Pérmico inferior a medio, el conjunto se halla recubierto por un "Verrucano" cuarcítico (véase 


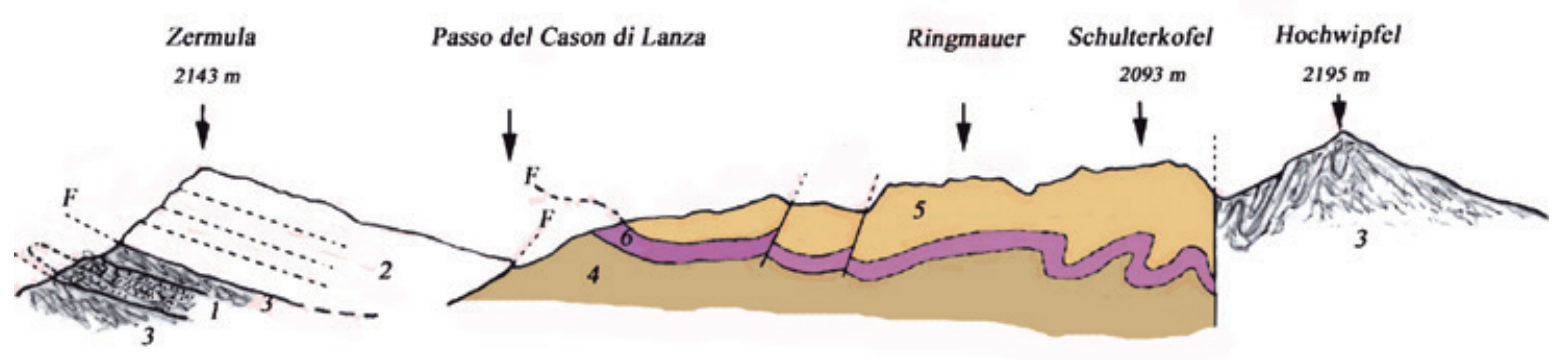

Figura 10. Sección a través de las estructuras. 1 : calizas rojas del Devónico inferior 2 : Devónico arrecifal del Monte Zermula 3 : Carbonífero inferior a medio del Hochwipfel 4 : Ámbito de Zollner See 5. Ámbito de Auernig 6 : Pérmico superior (Gröden y Bellerophon).

Figure 10. Cross-section. 1: Lower Devonian red limestone. 2 Non-reefal Devonian of Mount Zermula. 3. Lower to Mid-Carboniferous deposits of the Hochwipfel. 4. Zollner See Realm. 5. Auernig Realm. 6. Mid-Upper Permian (Gröden and Bellerophon).

también Cassinis et al. 2018). Incluso en la Isla de Elba se han citado fusulínidos de la misma edad (Bodechtel 1964; Kahler y Kahler 1969).

El ámbito de Zollner See podría aparecer mucho más lejos. Se adivina un amplio cinturón de terrenos en el contorno tethysiano "mesógeano" de las cadenas variscas, cuyas principales deformaciones son post-westfalienses y en las que el Carbonífero marino puede subir hasta el Namuriense $B$, incluso C o D (= Serpukhoviense-Bashkiriense). Se pueden asimismo mencionar, entre otros:

En la Toscana septentrional, en los Montes Pisani, se han indicado fósiles marinos (Pandelli et al. 2008) en el "Carbonífero superior" (?) de los "esquistos de San Lorenzo" que se hallan cubiertos por la brecha permiana de Ascianno, luego por el Verrucano del

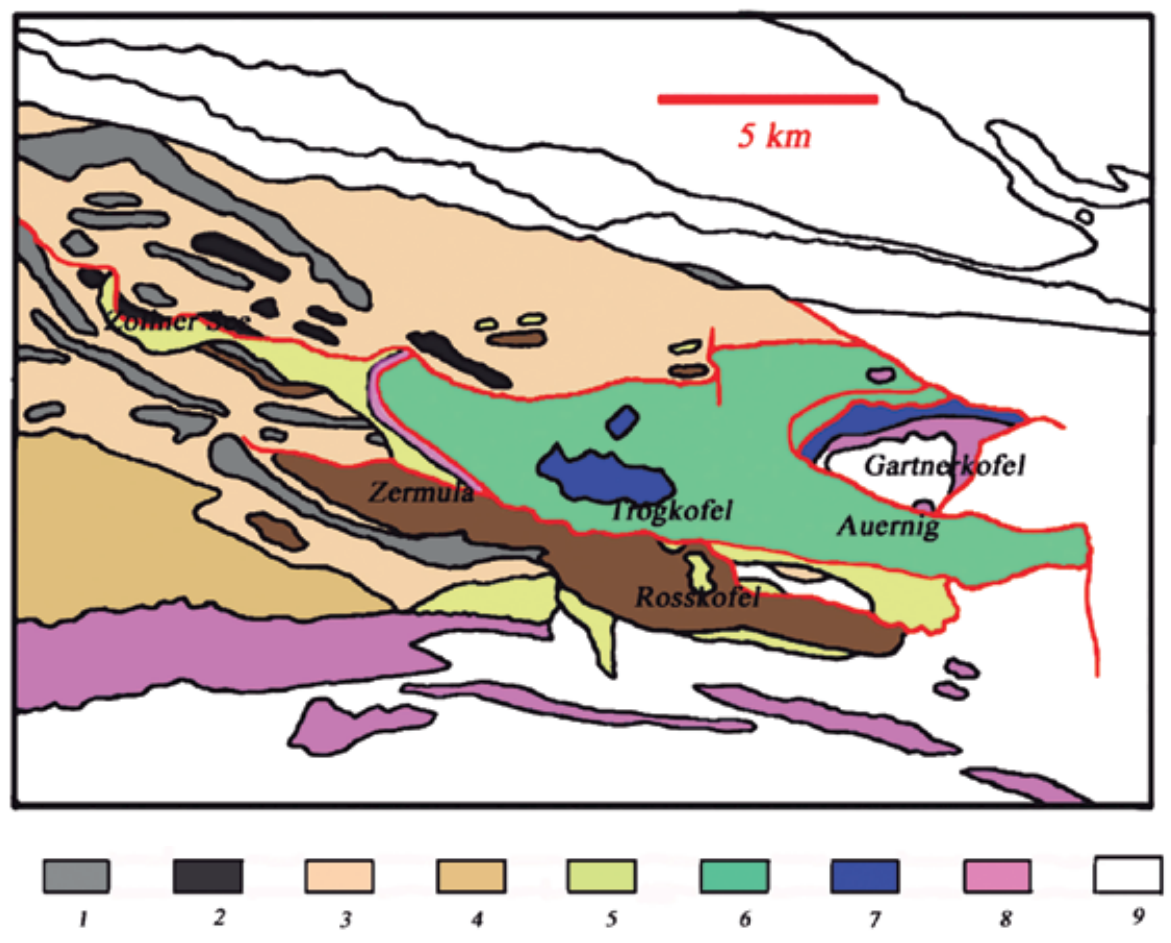

Figura 11. Esquema tectónico. 1 : Paleozoico inferior en general 2 : Liditas del Silúrico 3 : Carbonífero del Hochwipfel 4 : Carbonífero del Monte Dimón 5 : Ámbito de Zollner See 6 : Ámbito de Auernig 7 : Calizas deTrogkofel 8 : Pérmico superior (Gröden y Bellerophon) 9 . Triásico en general. Lineas rojas : contactos tectónicos.

Figure 11. Tectonic scheme. 1. Late Paleozoic in general. 2. Silurian Iydites. 3. Carboniferous of the Hochwipfel. 4. Carboniferous of the Mount Dimon. 5. Zollner See Realm. 6. Auernig Realm. 7. Trogkofel limestone. 8. Mid-Upper Permian (Gröden and Bellerophon). 9. Triassic in general. Red lines: Tectonic contacts. 
Ion Argyriadis y Daniel Vachard, 2020. Dos ámbitos paleogeográficos para el Paleozoico... Boletín Geológico y Minero, 131 (4): $795-815$

Monte della Verruca, de edad del Ladiniense? a Carniense. Aunque las dataciones, sobre todo en éste último, pueden ser discutibles.

En las Islas Baleares, donde, según la excelente síntesis de Bourrouilh (1983), se encuentran radiolaritas muy finas y niveles detríticos, sobre el Devónico, un Carbonífero inferior marino con calizas, sobre el que se sedimentó un Culm datado como Viseense superior a Namuriense B, gracias a conodontos, foraminíferos y goniatítidos, así como esporas y plantas.

Por supuesto, se conoce el Carbonífero de los Pirineos con su parte inferior marina y su Culm, así como la famosa "fauna de Mondette" cerca de Saint Girons, con Glyphioceras, que sube hasta el Namuriense (Delépine 1931). Tanto en los Pirineos como en las Islas Baleares, por encima del Carbonífero se encuentra en discordancia angular una potente serie roja. En la base de esta serie hay un conglomerado poligénico. El conglomerado es seguido por arcillas, pelitas rojas y areniscas grises o rojas. En Menorca se han separado polen y esporas del Pérmico superior (Bourrouilh 1983).

El ejemplo más claro y más completo de una serie que es casi perfectamente similar a la de Zollner See se encuentra en la Cordillera Cantábrica (véase Villa 1989; Villa et al. 2003; Sánchez de Posada et al. 1996; Villa y Sánchez de Posada 2009; etc.):

Un Carbonífero que va del Tournaisiense al Kasimoviense en una serie sin discordancia angular apreciable, en condiciones de tectónica tangencial importante, con superposiciones de unidades arrastradas, constituida por sedimentos (detríticos lutitas, areniscas, turbiditas) así como también calizas; el conjunto contiene fósiles marinos y también plantas. Los fósiles de origen marino son macrofósiles (braquiópodos, goniatítidos) para el Carbonífero inferior y fusulínidos para el Carbonífero superior (Villa et al. 2003). En toda la serie hay presencia de conodontos (cf. Sánchez de Posada et al. 1996). En la unidad de los Picos de Europa es donde la serie alcanza los niveles más elevados, con el Kasimoviense superior (Villa et al. 2003) e incluso, localmente, en la "formación de Puentellés" el Gzheliense basal (Villa y van Ginkel 2000; Villa y Ueno 2002). Las calizas de dicha formación contienen los géneros Montiparus, Rauserites, Jigulites, Tumefactus, probables Triticites, Ferganites y Quasifusulina (Villa et al. 2003). En todos los casos, el Pérmico detrítico rojo es transgresivo regionalmente sobre la serie del Carbonífero, erosionando el sustrato y con discordancia angular importante. Se percibe una gran similitud con la serie del ámbito de Zollner See. Por otra parte, los autores antes citados apuntan la correlación con los Alpes Cárnicos, sin constituir la distinción entre los dos ámbitos preponderantes que acabamos de establecer.

\section{Conclusiones}

Considerando el conjunto de datos expuestos a lo largo de este trabajo, se pueden definir la existencia de un ámbito fini-paleozoico que rodea el continente herciniano hacia la Mesogea post-herciniana. Se caracteriza por la existencia de un Paleozoico marino, más concretamente, de un Carbonífero marino que soporta descargas de origen terrestre con plantas $-y$ con carbón - que van subiendo hasta el Kasimoviense terminal, ocasionalmente hasta la base del Gzheliense, e incluso (Zollner See fide Heritsch 1936) hasta el Asseliense. El conjunto está marcado, al menos en los Alpes Cárnicos, por una esquistosidad verdadera de dirección $110^{\circ}$ y viene siendo transgredido por un Pérmico rojo en fuerte discordancia que, a su vez, evoluciona en los Alpes Cárnicos, hacia carbonatos y evaporitas, luego hacia un Triásico basal de facies Seis. Dicho ámbito se opone al de Auernig (Alpes Cárnicos) que no tiene sustrato conocido en las regiones estudiadas (es tectónicamente flotante, Figuras 8 y 9): empieza en el Gzheliense y la serie marina va subiendo -con descargas terrígenas, sin duda - al menos hasta el Pérmico medio con recurrencias de facies arrecifales. La transgresión de un Pérmico rojo mas fino se halla todavía presente, pero la discordancia saaliense es únicamente erosiva y el Pérmico terminal más calcáreo que evaporítico, con un Triásico marino mesogéano. Dicho ámbito, tal vez marcado de forma discontinua por los afloramientos dispersos y a veces dudosos de laToscana y de la Isla de Elba, se extiende francamente hacia el sudeste actual, en los Dinárides, las Helénides y las cadenas del sur de Anatolia, en todo lo que fue la Mesogea pérmica; es decir el "Paleotethys"y después el "Neotethys".

Tenemos pues:

Por una parte un ámbito (Zollner See) que bordea el edificio varisco, con la sedimentación marina que se interrumpe grosso modo en el techo del Carbonífero para retomarse, con líneas directrices totalmente diferentes en el Pérmico superior-Triásico inferior. Se supone que las cadenas variscas en plena gestación dibujaban ya entonces una orilla, que llamaremos proxi-tethysiana por razones semánticas, pero que las fases saaliense y asturiana han intervenido para integrar en la orogénesis de todo este cinturón.

Por otra parte, un ámbito (Auernig) cuyas facies marinas se extienden durante el Pérmico y regresan tras una interrupción poco significativa (incursiones de lutitas rojas a nivel de un Pérmico medio-superior). Dicho dominio es el de un mar epicontinental, limitado a la zona fótica (Argyriadis 1975, 1978), con numerosas formaciones arrecifales, lo que indica una apertura hacia el este (la tierra siempre ha girado en el mismo sentido, que implica que las aguas ricas en 
oxígeno empujadas por las corrientes subtropicales de la época se dirigían también en el mismo sentido). Se indica igualmente que no aparece ninguna traza de océano de la época entre Eurasia y Gondwana al menos hasta el Este de la Anatolia actual. Más allá, la situación cambia y la apertura tethysiana iniciada en el Triásico, se encuentra con una apertura que va del Ural hasta Madagascar, sellada por el Liásico (Argyriadis y Lys 1977).

Para concluir, cabe rendir homenaje al gran geólogo PierreTermier, quién, entre otros, fue el primero que vio y afirmó en los Alpes Cárnicos el corrimiento "de los Dinárides sobre los Alpídes". Dicho desplazamiento formaría parte de la orogénesis de edad cretácica (Argyriadis 1974, 2016) que Suess (1892), con una excelente intuición, Ilamó "Altaides póstumos". La cuestión que merece ser planteada es la de saber cuál es parte de esta orogénesis y de la varisca en el edificio de mantos de los Alpes Cárnicos.

\section{Agradecimientos}

Al Dr. Pedro Cózar Maldonado por su ayuda y su preciosa contribución. Al Dr. Juan Luis Alonso Alonso por sus preciosas sugerencias.

\section{Referencias}

Argyriadis, I. 1968. Le Permo-carbonifère des Alpes Carniques, jalon allochtone entre nord-alpin et sud-alpin. D.E.S., Travaux du Laboratoire de Géologie historique, Orsay, $156 \mathrm{pp}$.

Argyriadis, I. 1970. La position des Alpes Carniques dans l'orogène alpin et le problème de la limite alpino-dinarique. Bulletin de la Société géologique de France, (7), 12 (3), 473-480.

Argyriadis, I. 1974. Sur l'orogenèse mésogéenne des temps crétacés. Revue de Géographie Physique et Géologie Dynamique, 16 (1), 23-60.

Argyriadis, I. 1975. Mésogée permienne, chaîne hercynienne et cassure téthysienne. Bulletin de la Société géologique de France, (7), 17 (7), 56-67.

Argyriadis, I. 1978. Le Permien alpino-méditerranéen à la charnière entre I'Hercynien et I'Alpin. Thèse, Doctorat d'État, Université Paris-XI, Orsay, 491 pp.

Argyriadis, I. 2016. Las orogénesis superpuestas del edificio alpino-mediterráneo. Boletín Geológico y Minero, 127 (2/3), 593-612

Argyriadis, I. y Lys, M. 1977. Sur I'origine des continents mésozoïques : chaîne ouralienne, cassure téthysienne et cassure ouralo-malgache. Pour l'abandon du mythe de la Pangée. 5e Réunion Annuelle des Sciences de la Terre, Rennes, 13.
Balogh, K. 1964. A Bükk hegység földtani felépítése. Magyar Állami Földtani Intézet évkönyve, 48 (2), 555-705.

Bodechtel, J. 1964. Stratigraphie und Tektonik der Schuppenzone Elbas. Geologische Rundschau, 53, 25-41.

Bourrouilh, R. 1983. Estratigrafia, Sedimentologia y Tectónica de la Isla de Menorca y del Noreste de Mallorca (Baleares). La Terminación Nororiental de las Cordilleras béticas en el Mediterráneo occidental. Memorias del Instituto Geológico y Minero de España, 99, 672 pp.

Buggisch, W., Flügel, E., Leitz F. y Tietz, G.F. 1976. Die fazielle und paläogeographische Entwicklung im Perm der Karnischen Alpen und in den Randgebieten. Geologische Rundschau, 65, 649-690. https://doi.org/10.1007/ BF01808485

Cassinis, G., Avanzini, M., Cortesogno, L., Dallagiovanna, G., Di Stefano, P., Gaggero, L., Gullo, M., Massari, F., Neri, C., Ronchi, A., Seno, S. Vanossi, M. y Venturini, C. 1998. Synthetic Upper Paleozoic Correlation Charts of selected Italian areas. Atti Ticinensi di Scienze della Terra, 40, 65-120.

Cassinis, G., Perotti, C. y Santi, G. 2018. Post-Variscan Verrucano-like deposits in Italy, and the onset of the Alpine tectono-sedimentary cycle. Earth-Science Reviews, 185, 476-497.

Davydov, V.I., Krainer, K. y Chernykh, V. 2013. Fusulinid biostratigraphy of the Lower Permian Zweikofel Formation (Rattendorf Group; Carnic Alps, Austria) and Lower Permian Tethyan chronostratigraphy. Geological Journal, 48, 57-100. https://doi.org/10.1002/gj.2433

Delépine, G. 1931. L'âge des schistes de Mondette (Ariège). Comptes Rendus sommaires de la Société Géologique de France, 5 (1), 157-158.

Ellenberger, F. 1967. Les interférences de l'érosion et de la tectonique tangentielle tertiaire dans le Bas-Languedoc (principalement dans l'arc de St. Chinian); notes sur les charriages cisaillants. Revue de Géographie Physique et de Géologie dynamique, 2, IX, fasc. 2, 87-142.

Forke, H., Schönlaub, H.P.y Samankassou, E. 2006. Late Paleozoic of the Carnic Alps (Austria/ltaly). Field trip of the SCCS Task Group to estblish GSSP's close to the Moscovian/Kasimovian and Kasimovian/Gzhelian boundaries. July 31-August 1, 2006. Berichte der Geologischen Bundesanstalt, Wien, nr. 70, 57 pp.

Frech, F. 1894. Die Karnischen Alpen. Ein Beitrag zur vergleichenden Gebirgstektonik. Niemayer, Halle, 514 pp.

Gaertner, H.R. von 1931. Geologie der zentralkarnischen Alpen. Denkschriften der Österreischichen Akademie der Wissenschaften Wien, Mathematisch Naturwissenschaftliche Klasse, 102, 113-199.

Geyer, G. 1895. Aus dem palaeozoischen Gebiete der Karnischen Alpen. Verhandlungen der Geologischen Reichsanstalt, 1895 (2), 60-90.

Geyer, G. 1896. Die geologischen Verhältnisse im Pontafeler Abschnitt der Karnischen Alpen. Jahrbuch der Geologischen Reichsanstalt, 46, 127-233. 
Ion Argyriadis y Daniel Vachard, 2020. Dos ámbitos paleogeográficos para el Paleozoico... Boletín Geológico y Minero, 131 (4): $795-815$

Geyer, G. 1903. Exkursion in die Karnischen Alpen. Exkursionsführer zum IX. Internationalen Geologenkongress, Wien, $51 \mathrm{pp}$.

Geyer, G. 1911. Die Karnische Hauptkette der Südalpen. Geologischer Charakterbilder, Heft 9, Berlin, 6 Tafeln mit erläuterndem Text (6 tables with explicative text).

Gortani, M. 1906. Contribuzioni allo studio del Palaeozoico Carnico. I. La Fauna permocarbonifera del Col Mezzodi presso Forni Avoltri. Palaeontographia Italica, XII, 1-84.

Gortani, M. 1912. Rilevamento nel nucleo centrale Carnico. Bolletino del Real Comitato geológico d'ltalia, 43, 91-112.

Haas, J., Hámor, G. y Korpás, L. 1999. Geological setting and tectonic evolution of Hungary. Geologica Hungarica, series Geologica, 24, 179-196.

Heritsch, F. 1929. Die tektonische Stellung von Hochwipfelfazies und Nassfeldfazies des Karbons der Karnischen Alpen. Sitzungsberichte der Akademie der Wissenschaften in Wien, Abteilung I, 138, 413-423.

Heritsch, F. 1933. Die Stratigraphie von Oberkarbon und Perm in den Karnischen Alpen. Mitteilungen der geologischen Gesellschaft, 26, 162-190.

Heritsch, F. 1935. Neue Versteinerungen aus den Nassfeldschichten der Karnischen Alpen. Sitzungsberichte der Akademie der Wissenschaften in Wien, Abteilung I, Band 144, 349-375

Heritsch, F., 1936. Die Karnischen Alpen. Monographie einer Gebirgsgruppe der Ostalpen mit variszischem und alpidischem Bau. Geologisches Institut der Universität Graz Publisher, Graz, 295 pp.

Heritsch, F., Kahler, F. y Metz, K. 1934. Die Schichtenfolge von Oberkarbon und Unterperm. In: Heritsch, F. (ed.), Die Stratigraphie von Oberkarbon und Perm in den Karnischen Alpen. Mitteilungen der Geologischen Gesellschaft in Wien, 26 (1933), 163-180.

Kahler, F. 1932. Das Karbon der Rattendorfer Alm und des Nassfeldgebietes. Anzeiger der Akademie der Wissenschaften in Wien, I, 69, 241-243.

Kahler, F. 1934. Über das Vorkommen der Fusuliniden im Karbon und Perm der Karnischen Alpen. Anzeiger der Akademien der Wissenschaften in Wien, I, 71, 233-235.

Kahler, F. 1973. Beiträge zur Kenntnis der Fusuliniden der Ostalpen: Die Gattung Quasifusulina in den Karnischen Alpen. Palaeontographica, A, 141, 154-173.

Kahler, F. 1974. Fusuliniden aus T'ien-schan und Tibet. Mit Gedanken zur Geschichte der Fusuliniden-Meere im Perm. The Sino-Swedish Expedition, Publication 52, Volume nvertebrate Palaeontology, 4, 147 pp., Stockholm (The Sven Hedin Foundation).

Kahler, F. 1983. Fusuliniden aus dem Karbon und Perm der Karnischen Alpen und der Karawanken. Carinthia II, Sonderheft, 41, Klagenfurt, 107 pp.

Kahler, F. y Kahler, G. 1937. Beiträge zur Kenntnis der Fusuliniden der Ostalpen: Die Pseudoschwagerinen der
Grenzlandbänke und des oberen Schwagerinenkalkes. Palaeontographica, A, 87, 1-44.

Kahler, F. y Kahler, G. 1969. Einige südeuropäische Vorkommen von Fusuliniden. Mitteilungen der Geologischen Gesellschaft, 61 (1968), 40-60.

Kahler, F. y Krainer, K. 1993. The Schulterkofel Section in the Carnic Alps, Austria: Implications for the Carboniferous-Permian Boundary. Facies, 28, 257-276.

Kahler, F. y Prey, S. 1963. Erläuterungen zur geologischen Karte des Naßfeld-Gartnerkofel- Gebietes in den Karnischen Alpen. Geologische Bundesanstalt, Wien, 116 pp.

Krainer, K. 1992: Fazies, Sedimentationsprozesse und Paläogeographie im Karbon der Ost- und Südalpen. Jahrbuch der Geologischen Bundesanstalt, 135/1: 99-193, Wien.

Krainer, K. y Vachard, D. 2015. Late Visean (MFZ14) foraminifers and algae from the Kirchbach Limestone (Carnic Alps, Austria) and geological implications. Facies, 61, 418-441. http://www.springer.com/-/3/2b9b09c29ef94d1086800a90c0662fe9

Krainer, K., Sanders, D. y Schaffhauser, M. 2009. Early Permian shelf margin retreat and carbonate deposition, Zweikofel Massif, Carnic Alps (Austria). Austrian Journal of Earth Sciences, 102, 134-148.

Krainer, K., Vachard, D. y Schaffhauser, M. 2019a. Yakhtashian (Artinskian-Kungurian) cyanobacteria and calcareous algae from the Carnic Alps (Austria). Palaeontologia Electronica, 22.3.54A, 1-107. https://doi.org/10.26879/931.

Krainer, K., Vachard, D. y Schaffhauser, M. 2019b. Early Permian (Yakhtashian; Artinskian-early Kungurian) foraminifers and microproblematica from the Carnic Alps (Austria/ltaly). Abhandlungen der Geologischen Bundesanstalt, 73, $247 p p$.

Mariotti, A. 1972. Etudes stratigraphique et structurale d'une nouvelle série permo-carbonifère dans les Alpes Carniques : I'unité de Straniger Alm (Autriche). Conséquences pour la tectonique régionale, varisque et alpine. Bulletin de la Société géologique de France, (7), 14 (1), 25-33.

Pandeli, E., Dominici, S., Landi Degl'Innocenti, V., Cioppi, E. yTangocci, F. 2008. Marine fossils in the Late Carboniferous metasediments of the Pisani Mountains (Tuscany, Italy). In: Cassinis, G. (ed.), Stratigraphy and palaeogeography of late and post-Hercynian basins in the Southern Alps, Tuscany and Sardinia (Italy). Bollettino Società Geologica Italiana, 127 (3), 559-565.

Pasini, M. 1963. Alcuni Fusulinida della serie del Monte Auernig (Alpi Carniche) e loro significato stratigrafico. Rivista Italiana di Paleontologia, 69 (3), 337-382.

Pasini, M. 1991. Residual evidences of Permian carbonatic platform within the Apennine sequences (Italy). Bollettino della Società Geologica Italiana, 110, 843-848.

Pölser, P. 1969. Stratigraphie und Tektonik im Nordabfall des Findenigkofels (Silur bis Karbon; Karnischen Alpen, Österreich). Jahrbuch der Geologischen Bundesanstalt, 112, 355-398. 
Ion Argyriadis y Daniel Vachard, 2020. Dos ámbitos paleogeográficos para el Paleozoico... Boletín Geológico y Minero, 131 (4): $795-815$

Reichardt, W. 1937. Die ostalpinen Naßfeldschichten - eine Brücke zwischen Mitteleuropa und Rußland. Comptes Rendus 2 e Congrès Stratigraphie et Géologie du Carbonifère, Heerlen 1935, Maastricht, 2, 919-1055.

Richthofen, F. (von). 1860. Geognostische Beschreibung der Umgegend von Predazzo, Sanct Cassian und der Seisser Alpe in Süd-Tyrol. Mit einer geognostischen Karte und vier Profiltafeln. Justus Perthes, Gotha, XII, $328 p p$.

Sánchez de Posada, L.C., Martínez-Chacón, M.L., Mendez, C.A., Menéndez-Alvarez, J.R., Rios, L.M., Rodríguez, S., Truyols, J. y Villa, E. 1996. El Carbonífero marino del ámbito Astur-Leonés (Zona Cantábrica). Síntesis paleontológica. Revista Española de Paleontología, $n^{\circ}$ Extraordinario, 82-96.

Schellwien, E. 1892. Die Fauna des karnischen Fusulinidenkalks. Teil I. Palaeontographica 39, 1-56.

Schönlaub, H.P. 1985. Geologische Karte der Republik Österreich, Kötschach, 1:50.000. Geologische Bundesanstalt, Wien.

Schwinner, R. 1927. Die Schichtfolge des Seeberggebietes. Ein Vergleich. In: Heritsch, F. y Schwinner, R. (eds), Zur Geologie der Karnischen Alpen, III. Jahrbuch der Geologischen Bundesanstalt in Wien, 77 (1-2), 81-91.

Stache, G. 1872. Neue Fundstellen von Fusulinenkalk zwischen Gailthal und Canalthal in Kärnthen. Verhandlungen der kaiserlich-königlichen Geologischen Reichsanstalt, 283-287.

Suess, E. 1892. Das Antlitz der Erde. Erster Band. F. Tempsky, Wien, F. Tempsky, Prag, G. Freytag, Leipzig., 779 pp.

Szalai, T. 1979. A varisztikus északi és a Bükki tengeri felsðkarbon, Perm és Triász. Átalános Földtani Szemle, 12, 83-98.

Termier, P. 1922. Sur la structure des Alpes Orientales : Rapport des Dinarides et des Alpes. Comptes rendus de I'Académie des Sciences, 175, 1173-1178.

Vachard, D. 2016. Macroevolution and Biostratigraphy of Paleozoic Foraminifers. In: Montenari, M. (ed.): Stratigraphy \& Timescales, Elsevier, Amsterdam, 1, 257-323

Vachard, D. 2018. Permian smaller foraminifers; taxonomy, biostratigraphy and biogeography. In: Lucas, S.G. y Shen, S.Z. (eds.), The Permian Timescale. Geological Society of London, Special Publications, 450, 205-252.

Vachard, D. y Krainer, K. 2001a. Smaller foraminifers of the Upper Carboniferous Auernig Group, Carnic Alps (Aus-
tria/Italy). Rivista Italiana di Paleontologia e Stratigrafia, 107, 147-168.

Vachard, D. y Krainer, K. 2001b. Smaller foraminifers, characteristic algae and pseudo-algae of the latest Carboniferous/ Early Permian Rattendorf Group, Carnic Alps (Austria/Italy). Rivista Italiana de Paleontologia e Stratigrafia, 107, 169-195.

Vai, G.B. y Venturini, C. 1998. Moscovian and Artinskian rocks in the frame of the cyclic Permo- Carboniferous of the Carnic Alps and related areas. In: Crasquin Soleau, S. y De Wever, P. (eds.), Peri-Tethys: stratigraphic correlations. Geodiversitas, 19 (2), 173-186.

Venturini, C. 1990a. Geologia delle Alpi Carniche centro-orientali. Edizioni del Museo Friulano di Storia Naturale, Comune di Udine, 36, 217 pp.

Venturini, C. 1990b. Field Workshop on Carboniferous to Permian Sequence of the Pramollo- Nassfeld Basin (Carnic Alps). Guidebook, Arti Grafiche Friulane, Udine, 159 pp.

Villa, E. 1989. Fusulináceos carboníferos del este de Asturias (N. de España). Tesis doctoral, Universidad de Oviedo, $378 p p$.

Villa, E. y Ginkel, A. C. van 2000. Some late Moscovian and early Kasimovian fusulinaceans from the Las Llacerias section (Cantabrian Mountains, Spain). Journal of Foraminiferal Research, 30 (3), 219-243.

Villa, E. y Ueno, K. 2002. Characteristics and paleogeographic affinities of the early Gzhelian fusulinoideans from the Cantabrian Zone (NW Spain). Journal of Foraminiferal Research, 32(2), 135-154.

Villa, E., Merino-Tomé, O., Bahamonde, J.R. y Ueno, K. 2003. Fusulinoideans from the Puentellés Formation (Upper Carboniferous, NW Spain): Discussion on Phylogeny, Paleoecology and Paleogeography. Rivista Italiana de Paleontologia e Stratigrafia, 109, 2, 241 -253.

Villa, E. y Sánchez de Posada, L.C. 2009. Chapter 3, Carboniferous of the Cantabrian Zone. In: García Cortés, A. (ed.), Spanish Geological Frameworks and Geosites. An approach to Spanish geological heritage of international relevance. Instituto Geológico y Minero de España, 44-55.

Vinassa de Regny, P. 1910. Fossili ordoviciani del nucleo centrale carnico. Memorie dell'Academia Gioenia di Scienze Naturali di Catania, 5, 3, 48 pp.

Vinassa de Regny, P. y Gortani, M. 1905. Fossili carboniferi del Monte Pizzul e del Piano di Lanza nelle Alpi Carniche. Bolletino della Società Geologica Italiana, 24 (2), 461-520.

Recibido: julio 2019

Revisado: noviembre 2019

Aceptado: enero 2020

Publicado: marzo 2021 